EPHOU-98-003

April, 1998

\title{
Quantization of Even-Dimensional Actions of Chern-Simons Form with Infinite Reducibility
}

\author{
Noboru Kawamoto, Kazuhiko Suehiro, Takuya Tsukioka \\ AND HiRoshi Umetsu周 \\ Department of Physics, Hokkaido University \\ Sapporo, 060-0810, Japan
}

kawamoto, suehiro, tsukioka, umetsu@particle.sci.hokudai.ac.jp

\begin{abstract}
We investigate the quantization of even-dimensional topological actions of Chern-Simons form which were proposed previously. We quantize the actions by Lagrangian and Hamiltonian formulations à la Batalin, Fradkin and Vilkovisky. The models turn out to be infinitely reducible and thus we need an infinite number of ghosts and antighosts. The minimal actions of Lagrangian formulation which satisfy the master equation of Batalin and Vilkovisky have the same Chern-Simons form as the starting classical actions. In the Hamiltonian formulation we have used the formulation of cohomological perturbation and explicitly shown that the gauge-fixed actions of both formulations coincide even though the classical action breaks Dirac's regularity condition. We find an interesting relation that the BRST charge of Hamiltonian formulation is the odd-dimensional fermionic counterpart of the topological action of Chern-Simons form. Although the quantization of two dimensional models which include both bosonic and fermionic gauge fields are investigated in detail, it is straightforward to extend the quantization into arbitrary even dimensions. This completes the quantization of previously proposed topological gravities in two and four dimensions.
\end{abstract}

\footnotetext{
* Address after April 1998: Dept. of Phys. Osaka Univ. Toyonaka, Osaka 560-0043, Japan umetsu@phys.wani.osaka-u.ac.jp
} 


\section{Introduction}

It is obviously the most challenging problem how we can formulate the quantum gravity and the standard model in a unifying and constructive way. Toward a possible solution to this problem, the current trend is heading to the string related topics. It is, however, not obvious if the string is the only possible direction to this problem. In fact two-dimensional quantum gravity was formulated by a lattice gravity, the dynamical triangulation of random surface, equivalently by the matrix model. On the other hand three-dimensional Einstein gravity was successfully formulated by the Chern-Simons action even at the quantum level [1]. Since the Chern-Simons action is composed of one-form gauge field, the general covariance is automatic in the formulation.

If we combine the two successful formulations to find a new formulation of quantum gravity, we naturally lead to an idea that we should find a gauge theory formulated by all degrees of differential forms. Here each form corresponds to a fundamental simplex of a simplicial manifold. There are also good reasons that gravity theory can be formulated by a gauge theory.

The standard Chern-Simons action partly satisfies the above criteria. Previously one of the authors (N.K.) and Watabiki have proposed a new type of topological actions in arbitrary dimensions which have the Chern-Simons form [2]. The actions have the same algebraic structure as the ordinary Chern-Simons action and are formulated by all degrees of differential forms. It was shown that two-dimensional topological gravities [3] and a four-dimensional topological conformal gravity [1] were formulated by the evendimensional version of the generalized Chern-Simons actions at the classical level. It may not be an unnatural expectation that this type of formulation could play an important role in the formulation of quantum gravity.

Since the topological gravity theories mentioned above are defined at the classical level, it is natural to ask if they are well defined at the quantum level. In this paper we investigate the quantization of the models defined by the generalized Chern-Simons actions. In the analyses we don't specify an algebra in a particular way to accommodate some gravity theory. The stress should be on the quantization of the model itself. It turns out that the quantization of the generalized Chern-Simons action is highly nontrivial. There are two difficulties in quantizing these models. Firstly the action

has a zero form square term multiplied by the highest form and thus the vanishing condition of the zero form square is the equation of motion which breaks regularity 
condition [5, 6]. Secondly the theory is highly reducible, in fact infinitely reducible, as we show later.

In quantizing reducible systems we need to use the Lagrangian formulation developed by Batalin and Vilkovisky [7]. In order to clarify the role of the violation of Dirac's regularity condition we quantize the system in the Hamiltonian formulation of Batalin, Fradkin and Vilkovisky [8]. In the concrete analyses the quantization procedure of cohomological perturbation developed by Henneaux et al. [5] is used.

It was shown in the quantization of the simplest abelian version of generalized Chern-Simons action that the particular type of regularity violation does not cause serious problems for the quantization [6]. In this paper we investigate nonabelian version of Chern-Simons actions which turn out to be infinitely reducible while it was not the case for the abelian version. We show that the gauge-fixed action derived from the Lagrangian formulation leads to the same result of the Hamiltonian formulation even with regularity violating constraints and infinite reducibility.

In defining gauge fields and parameters, we introduce quaternion valued gauge fields and parameters containing forms of all possible degrees. They play an important role to manipulate the quantization in a unified manner. In other words the infinite number of ghost fields can be unified into a compact form and thus the quantization procedure can be largely simplified and becomes transparent even with the infinite reducibility. It is also interesting to note that the nonabelian version of the generalized Chern-Simons actions provide very fruitful and nontrivial examples for the quantization of infinitely reducible systems to be compared with Brink-Schwarz superparticle [9], Green-Schwarz superstring [10] and covariant string field theories [11].

This paper is organized as follows: We first briefly summarize the formulation of the generalized Chern-Simons actions in section 2. We then explain the quantization of two-dimensional generalized Chern-Simons model in the Lagrangian formulation in section 3. In section 4 the quantization of the same model is carried out by the Hamiltonian formulation. Section 5 explains the analyses of the perturbative aspects of the two-dimensional models. In section 6 we extend the quantization procedure of the two-dimensional model into arbitrary even dimensions, in particular we discuss a four-dimensional model as an example. Conclusions and discussions are given in the final section. 


\section{Generalization of Chern-Simons action into arbi- trary dimensions}

The generalized Chern-Simons actions, which were proposed by one of the authors (N.K.) and Watabiki some years ago, is a generalization of the ordinary three-dimensional Chern-Simons theory into arbitrary dimensions [2]. We summarize the results in this section. The essential point of the generalization is to extend a one-form gauge field and zero-form gauge parameter to a quaternion valued generalized gauge field and gauge parameter which contain forms of all possible degrees. Correspondingly the standard gauge symmetry is extended to much higher topological symmetry. These generalizations are formulated in such a way that the generalized actions have the same algebraic structure as the ordinary three-dimensional Chern-Simons action.

In the most general form, a generalized gauge field $\mathcal{A}$ and a gauge parameter $\mathcal{V}$ are defined by the following component form:

$$
\begin{aligned}
& \mathcal{A}=\mathbf{1} \psi+\mathbf{i} \hat{\psi}+\mathbf{j} A+\mathbf{k} \hat{A}, \\
& \mathcal{V}=\mathbf{1} \hat{a}+\mathbf{i} a+\mathbf{j} \hat{\alpha}+\mathbf{k} \alpha
\end{aligned}
$$

where $(\psi, \alpha),(\hat{\psi}, \hat{\alpha}),(A, a)$ and $(\hat{A}, \hat{a})$ are direct sums of fermionic odd forms, fermionic even forms, bosonic odd forms and bosonic even forms, respectively, and they take values on a gauge algebra. The bold face symbols $\mathbf{1}, \mathbf{i}, \mathbf{j}$ and $\mathbf{k}$ satisfy the algebra

$$
\begin{gathered}
\mathbf{1}^{2}=\mathbf{1}, \quad \mathbf{i}^{2}=\epsilon_{1} \mathbf{1}, \quad \mathbf{j}^{2}=\epsilon_{2} \mathbf{1}, \quad \mathbf{k}^{2}=-\epsilon_{1} \epsilon_{2} \mathbf{1}, \\
\mathbf{i j}=-\mathbf{j} \mathbf{i}=\mathbf{k}, \quad \mathbf{j} \mathbf{k}=-\mathbf{k} \mathbf{j}=-\epsilon_{2} \mathbf{i}, \quad \mathbf{k i}=-\mathbf{i} \mathbf{k}=-\epsilon_{1} \mathbf{j},
\end{gathered}
$$

where $\left(\epsilon_{1}, \epsilon_{2}\right)$ takes the value $(-1,-1),(-1,+1),(+1,-1)$ or $(+1,+1)$. Throughout this paper we adopt the convention $\left(\epsilon_{1}, \epsilon_{2}\right)=(-1,-1)$, then the above algebra corresponds to the quaternion algebra. The following graded Lie algebra can be adopted as a gauge algebra:

$$
\begin{gathered}
{\left[T_{a}, T_{b}\right]=f_{a b}^{c} T_{c},} \\
{\left[T_{a}, \Sigma_{\beta}\right]=g_{a \beta}^{\gamma} \Sigma_{\gamma},} \\
\left\{\Sigma_{\alpha}, \Sigma_{\beta}\right\}=h_{\alpha \beta}^{c} T_{c},
\end{gathered}
$$

where all the structure constants are subject to consistency conditions which follow from the graded Jacobi identities. If we choose $\Sigma_{\alpha}=T_{a}$ especially, this algebra reduces 
to $T_{a} T_{b}=k_{a b}^{c} T_{c}$ which is closed under multiplication. A specific example of such algebra is realized by Clifford algebra [3]. The components of the gauge field $\mathcal{A}$ and parameter $\mathcal{V}$ are assigned to the elements of the gauge algebra in a specific way:

$$
\begin{aligned}
& A=T_{a} A^{a}, \quad \hat{\psi}=T_{a} \hat{\psi}^{a}, \quad \psi=\Sigma_{\alpha} \psi^{\alpha}, \quad \hat{A}=\Sigma_{\alpha} \hat{A}^{\alpha}, \\
& \hat{a}=T_{a} \hat{a}^{a}, \quad \alpha=T_{a} \alpha^{a}, \quad \hat{\alpha}=\Sigma_{\alpha} \hat{\alpha}^{\alpha}, \quad a=\Sigma_{\alpha} a^{\alpha} .
\end{aligned}
$$

An element having the same type of component expansion as $\mathcal{A}$ and $\mathcal{V}$ belong to $\Lambda_{-}$ and $\Lambda_{+}$class, respectively, and these elements fulfill the following $Z_{2}$ grading structure:

$$
\left[\lambda_{+}, \lambda_{+}\right] \in \Lambda_{+}, \quad\left[\lambda_{+}, \lambda_{-}\right] \in \Lambda_{-}, \quad\left\{\lambda_{-}, \lambda_{-}\right\} \in \Lambda_{+},
$$

where $\lambda_{+} \in \Lambda_{+}$and $\lambda_{-} \in \Lambda_{-}$. The elements of $\Lambda_{-}$and $\Lambda_{+}$can be regarded as generalizations of odd forms and even forms, respectively. In particular the generalized exterior derivative which belongs to $\Lambda_{-}$is given by

$$
Q=\mathbf{j} d
$$

and the following relations similar to the ordinary differential algebra hold:

$$
\left\{Q, \lambda_{-}\right\}=Q \lambda_{-}, \quad\left[Q, \lambda_{+}\right]=Q \lambda_{+}, \quad Q^{2}=0,
$$

where $\lambda_{+} \in \Lambda_{+}$and $\lambda_{-} \in \Lambda_{-}$. To construct the generalized Chern-Simons actions, we need to introduce the two kinds of traces

$$
\begin{array}{ll}
\operatorname{Htr}\left[T_{a}, \cdots\right]=0, & \operatorname{Htr}\left[\Sigma_{\alpha}, \cdots\right]=0 \\
\operatorname{Str}\left[T_{a}, \cdots\right]=0, & \operatorname{Str}\left\{\Sigma_{\alpha}, \cdots\right\}=0,
\end{array}
$$

where $(\cdots)$ in the commutators or the anticommutators denotes a product of generators. In particular $(\cdots)$ should include an odd number of $\Sigma_{\alpha}$ 's in the last eq. of (2.7). These definitions of the traces are crucial to show that the generalized Chern-Simons action can be invariant under the generalized gauge transformation presented bellow.

After the above preparations, we can define four types of actions which have ChernSimons form,

$$
\begin{aligned}
S_{e}^{b} & =\int_{M} \operatorname{Htr}_{\mathbf{k}}\left(\frac{1}{2} \mathcal{A} Q \mathcal{A}+\frac{1}{3} \mathcal{A}^{3}\right), & S_{o}^{f} & =\int_{M} \operatorname{Htr}_{\mathbf{1}}\left(\frac{1}{2} \mathcal{A} Q \mathcal{A}+\frac{1}{3} \mathcal{A}^{3}\right), \\
S_{o}^{b} & =\int_{M} \operatorname{Str}_{\mathbf{j}}\left(\frac{1}{2} \mathcal{A} Q \mathcal{A}+\frac{1}{3} \mathcal{A}^{3}\right), & S_{e}^{f} & =\int_{M} \operatorname{Str}_{\mathbf{i}}\left(\frac{1}{2} \mathcal{A} Q \mathcal{A}+\frac{1}{3} \mathcal{A}^{3}\right),
\end{aligned}
$$

where $S_{e}^{b}, S_{o}^{f}, S_{o}^{b}$ and $S_{e}^{f}$ are an even-dimensional bosonic action, an odd-dimensional fermionic action, an odd-dimensional bosonic action and an even-dimensional fermionic 
action, respectively. $\operatorname{Htr}_{\mathbf{q}}(\cdots)$ and $\operatorname{Str}_{\mathbf{q}}(\cdots) \quad(\mathbf{q}=\mathbf{1}, \mathbf{i}, \mathbf{j}, \mathbf{k})$ are defined so as to pick up only the coefficients of $\mathbf{q}$ from $(\cdots)$ and take the traces defined by eq.(2.7). The reason why we obtain the four different types of action is related to the fact that the Chern-Simons term in the trace, $\frac{1}{2} \mathcal{A} Q \mathcal{A}+\frac{1}{3} \mathcal{A}^{3}$, belongs to $\Lambda_{-}$class and thus possesses the four different component types, the same types as in $\mathcal{A}$ of (2.1). We then need to pick up $d$-form terms to obtain $d$ dimensional actions defined on a manifold $M$. These actions are invariant up to surface terms under the generalized gauge transformation

$$
\delta \mathcal{A}=[Q+\mathcal{A}, \mathcal{V}]
$$

where $\mathcal{V}$ is the generalized gauge parameter defined by eq.(2.2). It should be noted that this symmetry is much larger than the usual gauge symmetry, in fact topological symmetry, since the gauge parameter $\mathcal{V}$ contains as many gauge parameters as gauge fields of various forms.

Substituting eqs.(2.1) and (2.2) into eqs.(2.8) and (2.9), we obtain explicit forms of the actions

$$
\begin{gathered}
S_{e}^{b}=-\int_{M} \operatorname{Htr}\left\{\hat{A}\left(d A+A^{2}+\hat{\psi}^{2}-\psi^{2}\right)+\frac{1}{3} \hat{A}^{3}+\psi(d \hat{\psi}+[A, \hat{\psi}])\right\}, \\
S_{o}^{f}=-\int_{M} \operatorname{Htr}\left\{\psi\left(d A+A^{2}+\hat{\psi}^{2}+\hat{A}^{2}\right)-\frac{1}{3} \psi^{3}-\hat{A}(d \hat{\psi}+[A, \hat{\psi}])\right\}, \\
S_{o}^{b}=-\int_{M} \operatorname{Str}\left\{\frac{1}{2} A d A+\frac{1}{3} A^{3}-\frac{1}{2} \hat{\psi}(d \hat{\psi}+[A, \hat{\psi}])+\hat{\psi}\{\psi, \hat{A}\}\right. \\
\left.-\frac{1}{2} \psi(d \psi+\{A, \psi\})-\frac{1}{2} \hat{A}(d \hat{A}+[A, \hat{A}])\right\}, \\
S_{e}^{f}=-\int_{M} \operatorname{Str}\left\{\hat{\psi}\left(d A+A^{2}-\psi^{2}+\hat{A}^{2}\right)+\frac{1}{3} \hat{\psi}^{3}+\hat{A}(d \psi+\{A, \psi\})\right\},
\end{gathered}
$$

and the gauge transformations

$$
\begin{aligned}
& \delta A=d \hat{a}+[A, \hat{a}]-\{\hat{\psi}, \alpha\}+[\psi, \hat{\alpha}]+\{\hat{A}, a\}, \\
& \delta \hat{\psi}=d \alpha+\{A, \alpha\}+[\hat{\psi}, \hat{a}]+[\psi, a]-\{\hat{A}, \hat{\alpha}\}, \\
& \delta \psi=-d \hat{\alpha}-[A, \hat{\alpha}]-[\hat{\psi}, a]+[\psi, \hat{a}]-[\hat{A}, \alpha], \\
& \delta \hat{A}=-d a-\{A, a\}+\{\hat{\psi}, \hat{\alpha}\}+[\psi, \alpha]+[\hat{A}, \hat{a}],
\end{aligned}
$$

where $[$,$] and \{$,$\} are commutator and anticommutator, respectively.$

Each action in (2.8) leads to the same equation of motion

$$
\mathcal{F}=Q \mathcal{A}+\mathcal{A}^{2}=0
$$


where $\mathcal{F}$ is a generalized curvature and thus the equation of motion is a vanishing curvature condition. Component expansions of the equations of motion are given by

$$
\begin{aligned}
d A+A^{2}+\hat{\psi}^{2}-\psi^{2}+\hat{A} & =0, \\
d \hat{\psi}+[A, \hat{\psi}]-\{\psi, \hat{A}\} & =0, \\
d \psi+\{A, \psi\}+[\hat{A}, \hat{\psi}] & =0, \\
d \hat{A}+[A, \hat{A}]+\{\hat{\psi}, \psi\} & =0 .
\end{aligned}
$$

We now show the explicit forms of two- and four-dimensional actions which will be used in this paper. We introduce the following notations:

$$
\begin{aligned}
\mathcal{A} & =\mathbf{1} \psi+\mathbf{i} \hat{\psi}+\mathbf{j} A+\mathbf{k} \hat{A} \\
& \equiv \mathbf{1}\left(\chi_{1}+\chi_{3}\right)+\mathbf{i}\left(\chi_{0}+\chi_{2}+\chi_{4}\right)+\mathbf{j}(\omega+\Omega)+\mathbf{k}(\phi+B+H)
\end{aligned}
$$

where $\phi, \omega, B, \Omega, H$ are bosonic $0-, 1-, 2-, 3-, 4$-form, and $\chi_{0}, \chi_{1}, \chi_{2}, \chi_{3}, \chi_{4}$ are fermionic 0-, 1-, 2-, 3-, 4-form, respectively. Substituting the above expressions into bosonic even action $S_{e}^{b}$ of $(2.10)$ and taking the two-form part, we obtain the twodimensional generalized Chern-Simons action

$$
S_{2}=-\int \operatorname{Htr}\left\{\phi\left(d \omega+\omega^{2}+\left\{\chi_{0}, \chi_{2}\right\}-\chi_{1}^{2}\right)+B\left(\phi^{2}+\chi_{0}^{2}\right)+\chi_{1}\left(d \chi_{0}+\left[\omega, \chi_{0}\right]\right)\right\} .
$$

Similarly the four-dimensional generalized Chern-Simons action can be obtained by taking the four-form part of the bosonic even action $S_{e}^{b}$ :

$$
S_{4}=-\int \operatorname{Htr}\left\{B\left(d \omega+\omega^{2}\right)+\phi\left(d \Omega+\{\omega, \Omega\}+B^{2}\right)+\phi^{2} H\right\}
$$

where we have omitted fermions for simplicity $(\psi=\hat{\psi}=0)$. Component wise explicit forms of gauge transformations and equations of motions for these actions can be obtained by eqs. (2.11) and (2.13) respectively and will be given later.

\section{Lagrangian quantization of two-dimensional mod- els}

\subsection{Infinite reducibility}

Hereafter we consider the even-dimensional bosonic action $S_{e}^{b}$ of (2.10), in particular the two-dimensional version (2.15) with a nonabelian gauge algebra as a concrete example although we will see that models in arbitrary even dimensions can be treated in the 
similar way. A simple example for nonabelian gauge algebras is given by Clifford algebra $c(0,3)$ generated by $\left\{T_{a}\right\}=\left\{1, i \sigma_{k} ; k=1,2,3\right\}$ where $\sigma_{k}$ 's are Pauli matrices [3]. Here we choose $\Sigma_{\alpha}=T_{a}$ for simplicity and thus the algebra is closed under multiplication. In this case the Htr satisfying the conditions in (2.7) reduces to the normal trace for matrices; Htr $\rightarrow$ Tr.

The quantization of a purely bosonic model, which is obtained by omitting fermionic gauge fields and parameters in the classical action and gauge transformations, was investigated in the previous paper [12]. Here we keep fermionic fields and thus investigate the most general model in two dimensions. Then the action expanded into components is given by

$$
\begin{aligned}
S_{0}=-\int d^{2} x \operatorname{Tr} \epsilon^{\mu \nu}\{ & \phi\left(\partial_{\mu} \omega_{\nu}+\omega_{\mu} \omega_{\nu}+\frac{1}{2}\left\{\chi, \chi_{\mu \nu}\right\}-\chi_{\mu} \chi_{\nu}\right) \\
& \left.+\frac{1}{2} B_{\mu \nu}\left(\phi^{2}+\chi^{2}\right)+\chi_{\mu}\left(\partial_{\nu} \chi+\left[\omega_{\nu}, \chi\right]\right)\right\},
\end{aligned}
$$

where $\epsilon^{01}=1$ and $\phi, \omega_{\mu}$ and $B_{\mu \nu}$ are scalar, vector and antisymmetric tensor fields while $\chi, \chi_{\mu}$ and $\chi_{\mu \nu}$ are fermionic fields of scalar, vector and antisymmetric tensor, respectively.* This Lagrangian possesses the following gauge symmetries corresponding to eq.(2.11):

$$
\begin{aligned}
\delta \phi= & {\left[\phi, v_{1}\right]-\left\{\chi, \xi_{1}\right\}, } \\
\delta \omega_{\mu}= & \partial_{\mu} v_{1}+\left[\omega_{\mu}, v_{1}\right]-\left\{\phi, u_{1 \mu}\right\}-\left[\chi_{\mu}, \xi_{1}\right]-\left\{\chi, \xi_{1 \mu}\right\}, \\
\delta B= & \epsilon^{\mu \nu}\left(\partial_{\mu} u_{1 \nu}+\left[\omega_{\mu}, u_{1 \nu}\right]\right)+\left[B, v_{1}\right]+\left[\phi, b_{1}\right] \\
& +\epsilon^{\mu \nu}\left\{\chi_{\mu}, \xi_{1 \nu}\right\}-\left\{\tilde{\chi}, \xi_{1}\right\}-\left\{\chi, \tilde{\xi}_{1}\right\} \\
\delta \chi= & \left\{\phi, \xi_{1}\right\}+\left[\chi, v_{1}\right], \\
\delta \chi_{\mu}= & \partial_{\mu} \xi_{1}+\left[\omega_{\mu}, \xi_{1}\right]-\left[\phi, \xi_{1 \mu}\right]+\left[\chi_{\mu}, v_{1}\right]+\left[\chi, u_{1 \mu}\right], \\
\delta \tilde{\chi}= & \epsilon^{\mu \nu}\left(\partial_{\mu} \xi_{1 \nu}+\left[\omega_{\mu}, \xi_{1 \nu}\right]\right)+\left\{B, \xi_{1}\right\}+\left\{\phi, \tilde{\xi}_{1}\right\} \\
& -\epsilon^{\mu \nu}\left[\chi_{\mu}, u_{1 \nu}\right]+\left[\tilde{\chi}, v_{1}\right]+\left[\chi, b_{1}\right],
\end{aligned}
$$

where $B, b_{1}, \tilde{\chi}$ and $\tilde{\xi}_{1}$ are defined by $B \equiv \frac{1}{2} \epsilon^{\mu \nu} B_{\mu \nu}, b_{1} \equiv \frac{1}{2} \epsilon^{\mu \nu} b_{1 \mu \nu}, \tilde{\chi} \equiv \frac{1}{2} \epsilon^{\mu \nu} \chi_{\mu \nu}$ and $\tilde{\xi}_{1} \equiv \frac{1}{2} \epsilon^{\mu \nu} \xi_{1 \mu \nu}$, respectively. For the later convenience we put the suffix 1 for the gauge parameters. Equations of motion (2.13) for this model are given by

$$
\phi: \quad-\epsilon^{\mu \nu}\left(\partial_{\mu} \omega_{\nu}+\omega_{\mu} \omega_{\nu}-\chi_{\mu} \chi_{\nu}\right)-\{\phi, B\}-\{\chi, \tilde{\chi}\}=0,
$$

${ }^{*}$ Throughout this paper we impose $\phi^{\dagger}=-\phi, \omega_{\mu}^{\dagger}=-\omega_{\mu}, B_{\mu \nu}^{\dagger}=B_{\mu \nu}, \chi^{\dagger}=-\chi, \chi_{\mu}^{\dagger}=\chi_{\mu}$ and $\chi_{\mu \nu}^{\dagger}=\chi_{\mu \nu}$ to make the classical action hermitian. 


$$
\begin{aligned}
\omega_{\mu}: & -\epsilon^{\mu \nu}\left(\partial_{\nu} \phi+\left[\omega_{\nu}, \phi\right]+\left\{\chi_{\nu}, \chi\right\}\right)=0, \\
B: & -\left(\phi^{2}+\chi^{2}\right)=0, \\
\chi: & -\epsilon^{\mu \nu}\left(\partial_{\mu} \chi_{\nu}+\left[\omega_{\mu}, \chi_{\nu}\right]\right)-[\phi, \tilde{\chi}]-[B, \chi]=0, \\
\chi_{\mu}: & \epsilon^{\mu \nu}\left(\partial_{\nu} \chi+\left[\omega_{\nu}, \chi\right]-\left\{\phi, \chi_{\nu}\right\}\right)=0, \\
\tilde{\chi}: & -[\phi, \chi]=0,
\end{aligned}
$$

where we have taken the right derivative of the corresponding fields.

As in the case of the purely bosonic model [12], this system is infinitely on-shell reducible. The reducibility is easily shown by extending the proof given for the purely bosonic model. First we introduce generalized variables

$$
\begin{aligned}
\mathcal{V}_{2 n}= & \mathbf{1} \xi_{2 n \mu} d x^{\mu}+\mathbf{i}\left(\xi_{2 n}+\frac{1}{2} \xi_{2 n \mu \nu} d x^{\mu} \wedge d x^{\nu}\right) \\
& +\mathbf{j} u_{2 n \mu} d x^{\mu}+\mathbf{k}\left(v_{2 n}+\frac{1}{2} b_{2 n \mu \nu} d x^{\mu} \wedge d x^{\nu}\right) \in \Lambda_{-}, \\
\mathcal{V}_{2 n+1}= & \mathbf{1}\left(v_{2 n+1}+\frac{1}{2} b_{2 n+1 \mu \nu} d x^{\mu} \wedge d x^{\nu}\right)-\mathbf{i} u_{2 n+1 \mu} d x^{\mu} \\
& -\mathbf{j}\left(\xi_{2 n+1}+\frac{1}{2} \xi_{2 n+1 \mu \nu} d x^{\mu} \wedge d x^{\nu}\right)+\mathbf{k} \xi_{2 n+1 \mu} d x^{\mu} \in \Lambda_{+}, \\
& n=0,1,2, \cdots,
\end{aligned}
$$

where the variables with index 0 denotes classical fields in the Lagrangian: $v_{0} \equiv \phi$, $u_{0, \mu} \equiv \omega_{\mu}, b_{0} \equiv B, \xi_{0} \equiv \chi, \xi_{0, \mu} \equiv \chi_{\mu}$ and $\tilde{\xi}_{0} \equiv \tilde{\chi}$ and thus $\mathcal{V}_{0}=\mathcal{A}$. The variables with index 1 are the original gauge parameters as in eqs.(3.2) and those with $n(>1)$ become the $n$-th reducibility parameters. The minus signs in eq.(3.5) are chosen for the later convenience. Then the transformation of $\mathcal{V}_{n}$

$$
\delta \mathcal{V}_{n}=(-)^{n}\left[Q+\mathcal{A}, \mathcal{V}_{n+1}\right]_{(-)^{n+1}}, \quad n=0,1,2, \cdots,
$$

satisfies the on-shell relation

$$
\begin{aligned}
\delta\left(\delta \mathcal{V}_{n}\right) & =\left.\delta \mathcal{V}_{n}\right|_{\mathcal{V}_{n+1} \rightarrow \mathcal{V}_{n+1}+\delta \mathcal{V}_{n+1}}-\delta \mathcal{V}_{n} \\
& =(-)^{n}\left[Q+\mathcal{A}, \delta \mathcal{V}_{n+1}\right]_{(-)^{n+1}} \\
& =(-)^{n}\left[Q+\mathcal{A},(-)^{n+1}\left[Q+\mathcal{A}, \mathcal{V}_{n+2}\right]_{(-)^{n+2}}\right]_{(-)^{n+1}} \\
& =-\left[\mathcal{F}, \mathcal{V}_{n+2}\right] \\
& =0
\end{aligned}
$$

where we have used the equation of motion (2.12). In the above equations, $[,]_{(-)^{n}}$ is a commutator for odd $n$ and an anticommutator for even $n$. Since the transformation 
(3.6) for $n=0$ represents the gauge transformation, eq.(3.7) implies that the gauge transformation is infinitely reducible. We give explicit reducibility transformations in the component form for the later use:

$$
\begin{aligned}
\delta v_{n}= & {\left[\phi, v_{n+1}\right]_{(-)^{n+1}}+(-)^{n+1}\left\{\chi, \xi_{n+1}\right\} } \\
\delta u_{n \mu}= & \partial_{\mu} v_{n+1}+\left[\omega_{\mu}, v_{n+1}\right]-\left[\phi, u_{n+1 \mu}\right]_{(-)^{n}} \\
& +(-)^{n+1}\left[\chi_{\mu}, \xi_{n+1}\right]_{(-)^{n+1}}+(-)^{n+1}\left\{\chi, \xi_{n+1 \mu}\right\} \\
\delta b_{n}= & \epsilon^{\mu \nu}\left(\partial_{\mu} u_{n+1 \nu}+\left[\omega_{\mu}, u_{n+1 \nu}\right]\right)+\left[B, v_{n+1}\right]_{(-)^{n+1}}+\left[\phi, b_{n+1}\right]_{(-)^{n+1}} \\
& +(-)^{n} \epsilon^{\mu \nu}\left[\chi_{\mu}, \xi_{n+1 \nu}\right]_{(-)^{n}}+(-)^{n+1}\left\{\tilde{\chi}, \xi_{n+1}\right\}+(-)^{n+1}\left\{\chi, \tilde{\xi}_{n+1}\right\} \\
\delta \xi_{n}= & {\left[\phi, \xi_{n+1}\right]_{(-)^{n}}+(-)^{n}\left[\chi, v_{n+1}\right], } \\
\delta \xi_{n \mu}= & \partial_{\mu} \xi_{n+1}+\left[\omega_{\mu}, \xi_{n+1}\right]-\left[\phi, \xi_{n+1 \mu}\right]_{(-)^{n+1}} \\
& +(-)^{n}\left[\chi_{\mu}, v_{n+1}\right]_{(-)^{n+1}}+(-)^{n}\left[\chi, u_{n+1 \mu}\right], \\
\delta \tilde{\xi}_{n}= & \epsilon^{\mu \nu}\left(\partial_{\mu} \xi_{n+1 \nu}+\left[\omega_{\mu}, \xi_{n+1 \nu}\right]\right)+\left[B, \xi_{n+1}\right]_{(-)^{n}}+\left[\phi, \tilde{\xi}_{n+1}\right]_{(-)^{n}} \\
& +(-)^{n+1} \epsilon^{\mu \nu}\left[\chi_{\mu}, u_{n+1 \nu}\right]_{(-)^{n}}+(-)^{n}\left[\tilde{\chi}, v_{n+1}\right]+(-)^{n}\left[\chi, b_{n+1}\right], \\
& \quad n=1,2,3, \cdots
\end{aligned}
$$

It is also important to recognize that $v_{n}, u_{n \mu}, b_{n}$ are bosonic parameters while $\xi_{n}, \xi_{n, \mu}$, $\tilde{\xi}_{n}$ are fermionic parameters.

Actually the infinite on-shell reducibility is a common feature of generalized ChernSimons theories with nonabelian gauge algebras in arbitrary dimensions, which can be understood by the fact that (3.7) is the relation among the generalized gauge fields and parameters. Thus generalized Chern-Simons theories add another category of infinitely reducible systems to known examples like Brink-Schwarz superparticle [9], Green-Schwarz superstring [10 and covariant string field theories [11. It should be noted that this theory is infinitely reducible though it contains only a finite number of fields of finite rank antisymmetric tensors. Brink-Schwarz superparticle and GreenSchwarz superstring are the similar examples in the sense that they contain only a finite number of fields yet are infinitely reducible. In the present case the infinite reducibility is understood from the following facts; firstly, the highest form degrees of $\mathcal{V}_{n}$ is unchanged from that of $\mathcal{V}_{n-1}$ in eq.(3.6) since the generalized gauge field $\mathcal{A}$ contains the zero form gauge field $\phi$ and $\chi$, secondly, the generalized Chern-Simons actions possess the same functional form (2.8) as the ordinary Chern-Simons action and thus have the vanishing curvature condition as the equation of motion; $\mathcal{F}=0(2.12)$. 
Thus the equations in (3.6) representing the infinite reducibility have the same form at any stage $n$, except for the difference between commutators and anticommutators. Algebraically, the structure of infinite reducibility resembles that of string field theories of a Chern-Simons form.

Before closing this section, we compare the generalized Chern-Simons theory of the abelian $g l(1, \mathbf{R})$ algebra with the model of nonabelian algebra. In the abelian case commutators in the gauge transformations vanish while anticommutators remain. Furthermore the field $\tilde{\chi}$ disappears from the classical Lagrangian. Then we can consistently put all transformation parameters to be zero except for $v_{1}, u_{1 \mu}, v_{2}$ and $\xi_{1}$. Thus the abelian model can be treated as a first stage reducible system. In particular the purely bosonic abelian model was explicitly quantized as a first stage reducible system in the previous paper [6]. In nonabelian cases, however, infinite reducibility is a universal and inevitable feature of the generalized Chern-Simons theories.

\subsection{Minimal sector}

In this section we present a construction of the minimal part of quantized action based on the Lagrangian formulation given by Batalin and Vilkovisky [7].

In the construction of Batalin and Vilkovisky, ghosts, ghosts for ghosts and the corresponding antifields are introduced according to the reducibility of the theory. We denote a minimal set of fields by $\Phi^{A}$ which include classical fields and ghost fields, and the corresponding antifields by $\Phi_{A}^{*}$. If a field has ghost number $n$, its antifield has ghost number $-n-1$. Then a minimal action is obtained by solving the master equation

$$
\begin{aligned}
\left(S_{\text {min }}\left(\Phi, \Phi^{*}\right), S_{\text {min }}\left(\Phi, \Phi^{*}\right)\right) & =0 \\
(X, Y) & =\frac{\partial_{r} X}{\partial \Phi^{A}} \frac{\partial_{l} Y}{\partial \Phi_{A}^{*}}-\frac{\partial_{r} X}{\partial \Phi_{A}^{*}} \frac{\partial_{l} Y}{\partial \Phi^{A}}
\end{aligned}
$$

with the boundary conditions

$$
\begin{aligned}
& \left.S_{\min }\right|_{\Phi_{A}^{*}=0}=S_{0}, \\
& \left.\frac{\partial S_{\min }}{\partial \Phi_{a_{n}}^{*}}\right|_{\Phi_{A}^{*}=0}=Z_{a_{n+1}}^{a_{n}} \Phi^{a_{n+1}}, \quad n=0,1,2, \cdots
\end{aligned}
$$

where $S_{0}$ is the classical action and $Z_{a_{n+1}}^{a_{n}} \Phi^{a_{n+1}}$ represents the $n$-th reducibility transformation where the reducibility parameters are replaced by the corresponding ghost fields. In this notation, the relation with $n=0$ in eq.(3.12) corresponds to the gauge 
transformation. The BRST transformations of $\Phi^{A}$ and $\Phi_{A}^{*}$ are given by

$$
s \Phi^{A}=\left(\Phi^{A}, S_{\min }\left(\Phi, \Phi^{*}\right)\right), \quad s \Phi_{A}^{*}=\left(\Phi_{A}^{*}, S_{\min }\left(\Phi, \Phi^{*}\right)\right) .
$$

Eqs.(3.9) and (3.13) assure that the BRST transformation is nilpotent and the minimal action is invariant under the transformation. In usual cases, the master equation is solved order by order with respect to the ghost number. Instead of solving an infinite set of equations due to the infinite reducibility in the present case, we can obtain the solution of the master equation (3.9) by using the characteristics of generalized ChernSimons theory in which fermionic and bosonic fields, and odd and even forms, can be treated in a unified manner [12].

First we introduce infinite fields

$$
\begin{aligned}
& C_{n}^{B}, C_{n \mu}^{B}, \widetilde{C}_{n}^{B}=\frac{1}{2} \epsilon^{\mu \nu} C_{n \mu \nu}^{B}, \\
& C_{n}^{F}, C_{n \mu}^{F}, \widetilde{C}_{n}^{F}=\frac{1}{2} \epsilon^{\mu \nu} C_{n \mu \nu}^{F}, \quad n=0, \pm 1, \pm 2, \cdots, \pm \infty,
\end{aligned}
$$

where the fields with index $B$ and $F$ are bosonic and fermionic, respectively. The index $n$ indicates the ghost number of the field. The fields with ghost number 0 are the classical fields

$$
\begin{array}{ll}
C_{0}^{B}=\phi, & C_{0 \mu}^{B}=\omega_{\mu}, \quad \widetilde{C}_{0}^{B}=B \\
C_{0}^{F}=\chi, & C_{0 \mu}^{F}=\chi_{\mu}, \quad \widetilde{C}_{0}^{F}=\tilde{\chi} .
\end{array}
$$

It is seen from eqs.(3.2) and (3.8) that fields content for ghosts and ghosts for ghosts in the minimal set is completed in the sector for $n>0$ while the necessary degrees of freedom for antifields are saturated for $n<0$. We will later identify fields with negative ghost numbers as antifields. We now redefine a generalized gauge field $\widetilde{\mathcal{A}}$ in such a form of (2.1) as it contains these infinite fields according to their Grassmann parity and form degrees:

$$
\begin{aligned}
\widetilde{\mathcal{A}}=\mathbf{1} \psi & +\mathbf{i} \hat{\psi}+\mathbf{j} A+\mathbf{k} \hat{A} \in \Lambda_{-}, \\
\psi & =\sum_{n=-\infty}^{\infty} C_{n \mu}^{F} d x^{\mu} \\
\hat{\psi} & =\sum_{n=-\infty}^{\infty}\left(C_{n}^{F}+\frac{1}{2} C_{n \mu \nu}^{F} d x^{\mu} \wedge d x^{\nu}\right), \\
A & =\sum_{n=-\infty}^{\infty} C_{n \mu}^{B} d x^{\mu}, \\
\hat{A} & =\sum_{n=-\infty}^{\infty}\left(C_{n}^{B}+\frac{1}{2} C_{n \mu \nu}^{B} d x^{\mu} \wedge d x^{\nu}\right) .
\end{aligned}
$$


We then introduce a generalized action for $\widetilde{\mathcal{A}}$ as

$$
\begin{aligned}
\widetilde{S}=\int \operatorname{Tr}_{\mathbf{k}}^{0}\left(\frac{1}{2} \widetilde{\mathcal{A}} Q \widetilde{\mathcal{A}}+\frac{1}{3} \widetilde{\mathcal{A}}^{3}\right) \\
=-\int d^{2} x \operatorname{Tr}^{0} \sum_{n=-\infty}^{\infty}\left\{C _ { n } ^ { B } \left(\epsilon^{\mu \nu} \partial_{\mu} C_{-n \nu}^{B}\right.\right. \\
\left.\quad+\sum_{m=-\infty}^{\infty}\left(\epsilon^{\mu \nu} C_{m \mu}^{B} C_{-(m+n) \nu}^{B}+\left\{C_{m}^{F}, \widetilde{C}_{-(m+n)}^{F}\right\}-\epsilon^{\mu \nu} C_{m \mu}^{F} C_{-(m+n) \nu}^{F}\right)\right) \\
+\sum_{m=-\infty}^{\infty} \widetilde{C}_{n}^{B}\left(C_{m}^{F} C_{-(m+n)}^{F}+C_{m}^{B} C_{-(m+n)}^{B}\right) \\
\left.\quad-C_{n}^{F} \epsilon^{\mu \nu}\left(\partial_{\mu} C_{-n \nu}^{F}+\sum_{m=-\infty}^{\infty}\left[C_{m \mu}^{B}, C_{-(m+n) \nu}^{F}\right]\right)\right\}
\end{aligned}
$$

where the upper index 0 on Tr indicates to pick up only the part with ghost number 0 .

One of the great advantage of generalized Chern-Simons formulation is that the quaternion valued gauge field and parameter which include different degrees of forms can be treated as if they were single gauge field and parameter. Here we would like to identify the generalized action $\widetilde{S}$ with the minimal action itself. In order to obtain the similar algebraic structure as (3.13) for the quaternion valued generalized gauge field, we heuristically introduce the following generalized antibracket:

$$
(X, Y)_{\lambda, \mathbf{k}}=\frac{1}{2} \int \operatorname{Tr}_{\mathbf{k}}\left(\frac{\delta_{r} X}{\delta \mathcal{A}} \frac{\delta_{l} Y}{\delta \mathcal{A}_{\lambda}^{*}}-\frac{\delta_{r} X}{\delta \mathcal{A}_{\lambda}^{*}} \frac{\delta_{l} Y}{\delta \mathcal{A}}\right),
$$

where we define left-, right-functional derivative of the "antifield" $\mathcal{A}_{\lambda}^{*}$ by

$$
\begin{gathered}
\frac{\delta_{l} X}{\delta \mathcal{A}_{\lambda}^{*}} \equiv \frac{\delta_{l}(\mathbf{i} \lambda X)}{\delta \mathcal{A}}=-\mathbf{i} \lambda \frac{\delta_{l} X}{\delta \mathcal{A}}, \\
\frac{\delta_{r} X}{\delta \mathcal{A}_{\lambda}^{*}} \equiv \frac{\delta_{r}(\mathbf{i} \lambda X)}{\delta \mathcal{A}}=\mathbf{i} \lambda \frac{\delta_{r} X}{\delta \mathcal{A}}
\end{gathered}
$$

where $\lambda$ is a fermionic scalar parameter with ghost number -1 and thus the relation, $\{\mathcal{A}, \mathbf{i} \lambda\}=0$ with $\mathcal{A}, \mathbf{i} \lambda \in \Lambda_{-}$, should be understood. The role of $\mathbf{i} \lambda$ in the generalized antibracket could be understood as an analogy from the opposite Grassmann parity nature of antifields in the standard Batalin and Vilkovisky formulation.

In the following we need to use the generalized antibracket only for the two cases; i) both $X$ and $Y$ are functionals $\left(X=\int \operatorname{Tr}_{\mathbf{k}} f(\mathcal{A}), f(\mathcal{A}) \in \Lambda_{-}\right)$, ii) $X$ is a function $(X=f(\mathcal{A}))$ and $Y$ is a functional. In these cases it suffices to define the generalized functional derivative which satisfies the following two properties:

$$
\begin{aligned}
& \text { 1) } \frac{\delta_{l, r} X}{\delta \mathcal{A}}=\frac{\partial_{l, r} f(\mathcal{A})}{\partial \mathcal{A}} \quad \text { for } \quad X=\int \operatorname{Tr}_{\mathbf{k}} f(\mathcal{A}), \quad\left(f(\mathcal{A}) \in \Lambda_{-}\right), \\
& \text {2) } \int \operatorname{Tr}_{\mathbf{k}}\left(\frac{\delta_{l, r} f(\mathcal{A})}{\delta \mathcal{A}} g(\mathcal{A})\right)=\frac{\partial_{l, r} f(\mathcal{A})}{\partial \mathcal{A}} g(\mathcal{A}), \quad\left(f(\mathcal{A}), g(\mathcal{A}) \in \Lambda_{-}\right) .
\end{aligned}
$$


In particular eq.(3.22) implies

$$
\int \operatorname{Tr}_{\mathbf{k}}\left(\frac{\delta_{l, r} \mathcal{A}}{\delta \mathcal{A}} g(\mathcal{A})\right)=g(\mathcal{A}) \quad \text { for } \quad g(\mathcal{A}) \in \Lambda_{-}
$$

By using the above properties of the generalized antibracket, we can show that the generalized action given in eq.(3.16) is invariant under the following transformation which is reminiscent of BRST transformation (3.13),

$$
\delta_{\lambda} \widetilde{\mathcal{A}} \equiv(\widetilde{\mathcal{A}}, \widetilde{S})_{\lambda, \mathbf{k}}=-\widetilde{\mathcal{F}} \mathbf{i} \lambda
$$

where $\widetilde{\mathcal{F}}$ is the generalized curvature $(2.12)$ constructed out of $\widetilde{\mathcal{A}}$ and the fermionic parameter $\lambda$ with ghost number -1 . It should be understood that the same ghost number sectors must be equated in eq.(3.23). Since $\widetilde{\mathcal{F}}$ and $\mathbf{i} \lambda$ belong to $\Lambda_{+}$and $\Lambda_{-}$, respectively, their product in the right hand side of eq.(3.23) belongs to the same $\Lambda_{-}$ class as $\widetilde{\mathcal{A}}$. The invariance of the action $\widetilde{S}$ under the transformation (3.23) can be checked by the manipulation

$$
\begin{aligned}
\delta_{\lambda} \widetilde{S} & =(\widetilde{S}, \widetilde{S})_{\lambda, \mathbf{k}} \\
& =-\int \operatorname{Tr}_{\mathbf{k}}^{0}\left\{\left(Q \widetilde{\mathcal{A}}+\widetilde{\mathcal{A}}^{2}\right) \widetilde{\mathcal{F}} \mathbf{i} \lambda\right\} \\
& =\int \operatorname{Tr}_{\mathbf{j}}^{0}(\widetilde{\mathcal{F}} \widetilde{\mathcal{F}}) \cdot \lambda \\
& =\int \operatorname{Tr}_{\mathbf{j}}^{0}\left\{Q\left(\widetilde{\mathcal{A}} Q \widetilde{\mathcal{A}}+\frac{2}{3} \widetilde{\mathcal{A}}^{3}\right)\right\} \cdot \lambda \\
& =0,
\end{aligned}
$$

where the subscript $\mathbf{j}$ plays the similar role as the subscript $\mathbf{k}$, i.e., to pick up only the coefficient of $\mathbf{j}$ in the trace. The change of the subscript $\mathbf{k}$ to $\mathbf{j}$ is necessary to take $\mathbf{i}$ into account in the trace in accordance with $\mathbf{j i}=-\mathbf{k}$. Here we have simply ignored the boundary term and thus the invariance is valid up to the surface term.

We now show that a right variation $s$ defined by $\delta_{\lambda} \widetilde{\mathcal{A}}=s \widetilde{\mathcal{A}} \lambda$ is the BRST transformation. First of all this transformation is nilpotent:

$$
s^{2} \widetilde{\mathcal{A}} \lambda_{2} \lambda_{1} \equiv \delta_{\lambda_{2}} \delta_{\lambda_{1}} \widetilde{\mathcal{A}}=-\delta_{\lambda_{2}} \widetilde{\mathcal{F}} \mathbf{i} \lambda_{1}=-[Q+\widetilde{\mathcal{A}}, \widetilde{\mathcal{F}}] \lambda_{2} \lambda_{1}=0
$$

where the generalized Bianchi identity is used

$$
[Q+\widetilde{\mathcal{A}}, \tilde{\mathcal{F}}]=\left[Q+\widetilde{\mathcal{A}},(Q+\widetilde{\mathcal{A}})^{2}\right]=0
$$

Next we need to show that the transformation $s$ is realized as the antibracket form of (3.13). The invariance of $\widetilde{S}$ under (3.23) implies that $\widetilde{S}$ is indeed the minimal action 
if we make a proper identification of fields of negative ghost numbers with antifields. It is straightforward to see that the BRST transformations (3.13), both for fields and antifields, are realized under the following identifications with $S_{\min }=\widetilde{S}$ :

$$
\begin{aligned}
& C_{-n \mu}^{F}=\epsilon_{\mu \nu}^{-1} C_{n-1}^{B \nu *}, \quad C_{-n \mu}^{B}=\epsilon_{\mu \nu}^{-1} C_{n-1}^{F \nu *}, \\
& C_{-n}^{F}=\widetilde{C}_{n-1}^{B *}, \quad C_{-n}^{B}=-\widetilde{C}_{n-1}^{F *}, \\
& \widetilde{C}_{-n}^{F}=C_{n-1}^{B *}, \quad \widetilde{C}_{-n}^{B}=-C_{n-1}^{F *}, \quad n=1,2,3, \cdots,
\end{aligned}
$$

where $\epsilon_{\mu \nu}^{-1}$ is the inverse of $\epsilon^{\mu \nu}, \epsilon^{\mu \rho} \epsilon_{\rho \nu}^{-1}=\delta_{\nu}^{\mu}{ }^{\dagger}$ This shows that we have obtained a solution for the master equation (3.9):

$$
\delta_{\lambda} S_{\min }=\left(S_{\min }, S_{\min }\right)_{\lambda, \mathbf{k}}=\left(S_{\min }, S_{\min }\right) \cdot \lambda=0
$$

where $($,$) is the original antibracket defined by (3.10).$

It is easy to see that this solution satisfies the boundary conditions (3.11) and (3.12), by comparing the gauge transformations (3.2) and the reducibilities (3.8) with the following expansion of $S_{\min }$ :

$$
\begin{aligned}
S_{\min }=S_{0}+ & \int d^{2} x \operatorname{Tr}\left\{\sum _ { n = 0 } ^ { \infty } \left\{C_{n}^{B *}\left(\left[\phi, C_{n+1}^{F}\right]-\left[\chi, C_{n+1}^{B}\right]\right)+C_{n}^{F *}\left(\left\{\phi, C_{n+1}^{B}\right\}+\left\{\chi, C_{n+1}^{F}\right\}\right)\right.\right. \\
& +C_{n}^{B \mu *}\left(\partial_{\mu} C_{n+1}^{F}+\left[\omega_{\mu}, C_{n+1}^{F}\right]-\left\{\phi, C_{n+1 \mu}^{F}\right\}-\left\{\chi_{\mu}, C_{n+1}^{B}\right\}-\left[\chi, C_{n+1 \mu}^{B}\right]\right) \\
& +C_{n}^{F \mu *}\left(\partial_{\mu} C_{n+1}^{B}+\left[\omega_{\mu}, C_{n+1}^{B}\right]-\left[\phi, C_{n+1 \mu}^{B}\right]+\left\{\chi_{\mu}, C_{n+1}^{F}\right\}+\left\{\chi, C_{n+1 \mu}^{F}\right\}\right) \\
& +\widetilde{C}_{n}^{B *}\left(\epsilon^{\mu \nu}\left(\partial_{\mu} C_{n+1 \nu}^{F}+\left[\omega_{\mu}, C_{n+1 \nu}^{F}\right]+\left[\chi_{\mu}, C_{n+1 \nu}^{B}\right]\right)\right. \\
& \left.\quad+\left[B, C_{n+1}^{F}\right]+\left[\phi, \widetilde{C}_{n+1}^{F}\right]-\left[\tilde{\chi}, C_{n+1}^{B}\right]-\left[\chi, \widetilde{C}_{n+1}^{B}\right]\right) \\
& +\widetilde{C}_{n}^{F *}\left(\epsilon^{\mu \nu}\left(\partial_{\mu} C_{n+1 \nu}^{B}+\left[\omega_{\mu}, C_{n+1 \nu}^{B}\right]-\left[\chi_{\mu}, C_{n+1 \nu}^{F}\right]\right)\right. \\
& \left.\left.\left.\quad+\left\{B, C_{n+1}^{B}\right\}+\left\{\phi, \widetilde{C}_{n+1}^{B}\right\}+\left\{\tilde{\chi}, C_{n+1}^{F}\right\}+\left\{\chi, \widetilde{C}_{n+1}^{F}\right\}\right)\right\}+\cdots \cdots\right\} .
\end{aligned}
$$

Thus the action $S_{\min }=\widetilde{S}$ with the identification $(3.25)$ is the correct solution of the master equation for the generalized Chern-Simons theory. The signs in eq.(3.5) have been chosen so that the boundary conditions are satisfied without additional signs in the definition of ghost fields in eq.(3.15).

For completeness we give explicit forms of the BRST transformations of the minimal fields

$$
s C_{n}^{B}=-\sum_{m=-\infty}^{\infty}\left[C_{m}^{F}, C_{n-m+1}^{B}\right],
$$

\footnotetext{
$\dagger$ To be precise the antifields are defined as $C_{n}^{B *}=C_{n a}^{B *}\left(\eta^{-1}\right)^{a b} T_{b}, \cdots$, with $\operatorname{Tr} T_{a} T_{b}=\eta_{a b}$.
} 


$$
\begin{aligned}
& s C_{n}^{F}=\sum_{m=-\infty}^{\infty}\left(\frac{1}{2}\left\{C_{m}^{B}, C_{n-m+1}^{B}\right\}+\frac{1}{2}\left\{C_{m}^{F}, C_{n-m+1}^{F}\right\}\right), \\
& s C_{n \mu}^{B}=\partial_{\mu} C_{n+1}^{F}+\sum_{m=-\infty}^{\infty}\left(\left[C_{m \mu}^{B}, C_{n-m+1}^{F}\right]-\left\{C_{m \mu}^{F}, C_{n-m+1}^{B}\right\}\right), \\
& s C_{n \mu}^{F}=\partial_{\mu} C_{n+1}^{B}+\sum_{m=-\infty}^{\infty}\left(\left[C_{m \mu}^{B}, C_{n-m+1}^{B}\right]+\left\{C_{m \mu}^{F}, C_{n-m+1}^{F}\right\}\right), \\
& s \widetilde{C}_{n}^{B}=\epsilon^{\mu \nu} \partial_{\mu} C_{n+1 \nu}^{F}+\sum_{m=-\infty}^{\infty}\left(\epsilon^{\mu \nu}\left[C_{m \mu}^{B}, C_{n-m+1 \nu}^{F}\right]\right. \\
& s \widetilde{C}_{n}^{F}=\epsilon^{\mu \nu} \partial_{\mu} C_{n+1 \nu}^{B}+\sum_{m=-\infty}^{\infty}\left(\frac{1}{2} \epsilon^{\mu \nu}\left[C_{m \mu}^{B}, C_{n-m+1 \nu}^{B}, C_{n-m+1}^{B}\right]-\left[C_{m}^{F}, \widetilde{C}_{n-m+1}^{B}\right]\right), \\
& \left.\quad+\left\{C_{m}^{B \nu}, \widetilde{C}_{n-m+1}^{B}\right\}+\left\{C_{m \mu}^{F}, C_{n-m+1 \nu}^{F}, \widetilde{C}_{n-m+1}^{F}\right\}\right),
\end{aligned}
$$

where the identification (3.25) should be understood.

It is critical in our construction of the minimal action that the action of the generalized theory possesses the same structure as the Chern-Simons action and fermionic and bosonic fields are treated in a unified manner. It is interesting to note that the starting classical action and the minimal action which includes the infinite series of bosonic and fermionic fields have the same form of $(2.8)$ with the replacement $\mathcal{A} \rightarrow \widetilde{\mathcal{A}}$. This is reminiscent of string field theories whose actions have the Chern-Simons form: a string field contains infinite series of ghost fields and antifields. The minimal action also takes the same Chern-Simons form [11]. It is also worth mentioning that there are other examples where classical fields and ghost fields are treated in a unified way 13.

It is obvious that the minimal action for generalized Chern-Simons theory in arbitrary even dimensions can be constructed in the same way as in the two-dimensional case because the classical action (2.8), gauge symmetries (2.9), reducibility transfor-

mations (3.6), the minimal action (3.16) and BRST transformations $s \widetilde{\mathcal{A}}=-\widetilde{\mathcal{F}} \mathbf{i}$ are described by using generalized fields and parameters.

\subsection{Gauge-fixed action}

The gauge degrees of freedom are fixed by introducing a nonminimal action which must be added to the minimal one, and choosing a suitable gauge fermion. Though the number of gauge-fixing conditions is determined in accordance with the "real" gauge degrees of freedom, we can prepare a redundant set of gauge-fixing conditions and then compensate the redundancy by introducing extraghosts. Indeed Batalin and Vilkovisky 
gave a general prescription to construct a nonminimal sector by this procedure [7]. This prescription is, however, inconvenient in the present case since it leads to a doubly infinite number of fields; antighosts, extraghosts, $\cdots$, where "doubly infinite" means the infinities both in the vertical direction and the horizontal direction in the triangular tableau of ghosts. In the case of the purely bosonic model, we could find gauge-fixing conditions so that such extra infinite series do not appear while propagators for all fields be well-defined. This type of gauge-fixing prescription which is unconventional for the Batalin-Vilkovisky formulation is known, for example, in a quantization of topological Yang-Mills theory [14, 15]. Those gauge-fixing conditions are easily extended to the present case and we can adopt the standard Landau type gauge-fixing for the vector and antisymmetric tensor fields in each sector of the ghost number, which is sufficient to make a complete gauge-fixing.

We introduce the nonminimal action

$$
\begin{aligned}
S_{\text {nonmin }}=\int d^{2} x \sum_{n=1}^{\infty} \operatorname{Tr}( & \bar{C}_{n}^{F *} b_{n-1}^{B}+\bar{C}_{n}^{B *} b_{n-1}^{F}+\bar{C}_{n \mu}^{F *} b_{n-1}^{B \mu}+\bar{C}_{n \mu}^{B *} b_{n-1}^{F \mu} \\
& \left.+\eta_{n-1}^{F *} \pi_{n}^{B}+\eta_{n-1}^{B *} \pi_{n}^{F}\right)
\end{aligned}
$$

where the ghost number of nonminimal fields is $n$ for $\eta_{n}^{B, F}, \pi_{n}^{B, F}$ and $-n$ for $\bar{C}_{n}^{B, F}$, $\bar{C}^{B, F \mu}, b_{n}^{B, F}$, and the corresponding antifields possess ghost number $-n-1$ and $n-1$, respectively. The indices $B$ and $F$ represent the Grassmannian property of fields as before. The BRST transformations of these fields are defined by this nonminimal action,

$$
\begin{array}{rlrl}
s \bar{C}_{n}^{F, B} & =b_{n-1}^{B, F}, & s b_{n-1}^{B, F}=0, & \\
s \bar{C}_{n}^{F, B \mu}=b_{n-1}^{B, F \mu}, & s b_{n-1}^{B, F \mu}=0, & \\
s \eta_{n-1}^{F, B}=\pi_{n}^{B, F}, & s \pi_{n}^{B, F}=0, & \\
s \bar{C}_{n}^{F, B *}=0, & s b_{n-1}^{B *}=-\bar{C}_{n}^{F *}, & s b_{n-1}^{F *}=\bar{C}_{n}^{B *}, \\
s \bar{C}_{n \mu}^{F, B *}=0, & s b_{n-1 \mu}^{B *}=-\bar{C}_{n \mu}^{F *}, & s b_{n-1 \mu}^{F *}=\bar{C}_{n \mu}^{B *}, \\
s \eta_{n-1}^{F, B *}=0, & s \pi_{n}^{B *}=-\eta_{n-1}^{F *}, & s \pi_{n}^{F *}=\eta_{n-1}^{B *} .
\end{array}
$$

The suffix $F, B$, which denotes fermionic or bosonic property of each ghost fields, represents both relations with the given order. Next we adopt the following gauge fermion $\Psi$ which leads to a Landau type gauge-fixing,

$$
\begin{array}{r}
\Psi=\int d^{2} x \sum_{n=1}^{\infty} \operatorname{Tr}\left(\bar{C}_{n}^{B} \partial^{\mu} C_{n-1 \mu}^{F}+\bar{C}_{n}^{F} \partial^{\mu} C_{n-1 \mu}^{B}+\bar{C}_{n}^{B \mu} \epsilon_{\mu \nu}^{-1} \partial^{\nu} \widetilde{C}_{n-1}^{F}\right. \\
\left.+\bar{C}_{n}^{F \mu} \epsilon_{\mu \nu}^{-1} \partial^{\nu} \widetilde{C}_{n-1}^{B}+\bar{C}_{n}^{B \mu} \partial_{\mu} \eta_{n-1}^{F}+\bar{C}_{n}^{F \mu} \partial_{\mu} \eta_{n-1}^{B}\right)
\end{array}
$$


where we assume a flat metric for simplicity. Then the antifields can be eliminated by equations $\Phi_{A}^{*}=\frac{\partial \Psi}{\partial \Phi^{A}}$

$$
\begin{array}{llrl}
C_{n}^{F, B *}=0, & C_{n}^{F, B \mu *}=-\partial^{\mu} \bar{C}_{n+1}^{B, F}, \\
\widetilde{C}_{n}^{F, B *}=\epsilon_{\mu \nu}^{-1} \partial^{\mu} \bar{C}_{n+1}^{B, F \nu}, & \bar{C}_{n+1}^{F, B *}=\partial^{\mu} C_{n \mu}^{B, F}, \\
\bar{C}_{n+1 \mu}^{F, B *}=\epsilon_{\mu \nu}^{-1} \partial^{\nu} \widetilde{C}_{n}^{B, F}+\partial_{\mu} \eta_{n}^{B, F}, & \eta_{n-1}^{F, B *}=-\partial_{\mu} \bar{C}_{n}^{B, F \mu},
\end{array}
$$

$$
n=0,1,2, \cdots
$$

The complete gauge-fixed action $S_{t o t}$ is

$$
S_{t o t}=\left.S_{\min }\right|_{\Sigma}+\left.S_{\text {nonmin }}\right|_{\Sigma}
$$

where $\Sigma$ is a surface defined by eq.(3.31). This action is invariant under the on-shell nilpotent BRST transformations (3.27) and (3.29) in which the antifields are eliminated by substituting eqs.(3.31). It can be seen that the propagators of all fields are welldefined, by writing the kinetic terms and the gauge-fixing terms in

$$
\begin{aligned}
S_{\text {tot }}=\int d^{2} x \operatorname{Tr}\{ & -\phi \epsilon^{\mu \nu} \partial_{\mu} \omega_{\nu}+\partial^{\mu} \omega_{\mu} b_{0}^{B}+\epsilon_{\mu \nu}^{-1} \partial^{\nu} B b_{0}^{B \mu} \\
& +\chi \epsilon^{\mu \nu} \partial_{\mu} \chi_{\nu}+\partial^{\mu} \chi_{\mu} b_{0}^{F}+\epsilon_{\mu \nu}^{-1} \partial^{\nu} \tilde{\chi} b_{0}^{F \mu} \\
& +\sum_{G=F, B} \sum_{n=1}^{\infty}\left(-\partial^{\mu} \bar{C}_{n}^{G} \partial_{\mu} C_{n}^{G}-\frac{1}{2}\left(\partial^{\mu} \bar{C}_{n}^{G \nu}-\partial^{\nu} \bar{C}_{n}^{G \mu}\right)\left(\partial_{\mu} C_{n \nu}^{G}-\partial_{\nu} C_{n \mu}^{G}\right)\right) \\
& +\sum_{G=F, B} \sum_{n=1}^{\infty}\left(\partial^{\mu} C_{n \mu}^{G} b_{n}^{G}+\epsilon_{\mu \nu}^{-1} \partial^{\nu} \widetilde{C}_{n}^{G} b_{n}^{G \mu}+\partial_{\mu} \eta_{n-1}^{G} b_{n-1}^{G \mu}-\partial_{\mu} \bar{C}_{n}^{G \mu} \pi_{n}^{G}\right) \\
& + \text { interaction terms }\} .
\end{aligned}
$$

Thus the gauge fermion (3.30) is a correct choice and the gauge degrees of freedom are fixed completely. We can consistently determine the hermiticity of the fields with a convention $\lambda^{\dagger}=-\lambda$ in eq. 3.23 )

Here comes a possible important comment. There is a common feature for models of infinitely reducible systems. When the number of reducibility parameters at each level is the same as that of gauge parameters, the number of the "real" gauge degrees

\footnotetext{
$\ddagger$ Hermiticity conditions;

$$
\begin{aligned}
& C_{n}^{F, B \dagger}=-C_{n}^{F, B}, \quad C_{n \mu}^{F \dagger}=C_{n \mu}^{F}, \quad C_{n \mu}^{B \dagger}=-C_{n \mu}^{B}, \quad \widetilde{C}_{n}^{F, B \dagger}=\widetilde{C}_{n}^{F, B}, \\
& \bar{C}_{n}^{F \dagger}=\bar{C}_{n}^{F}, \quad \bar{C}_{n}^{B \dagger}=-\bar{C}_{n}^{B}, \quad \bar{C}_{n}^{F, B \mu \dagger}=-\bar{C}_{n}^{F, B \mu}, \\
& b_{n}^{F, B \dagger}=-b_{n}^{F, B}, \quad b_{n}^{F \mu \dagger}=-b_{n}^{F \mu}, \quad b_{n}^{B \mu \dagger}=-b_{n}^{B \mu}, \quad \eta_{n}^{F, B \dagger}=\eta_{n}^{F, B}, \quad \pi_{n}^{F \dagger}=\pi_{n}^{F} \quad \pi_{n}^{B \dagger}=-\pi_{n}^{B} .
\end{aligned}
$$
}


of freedom is the half of the original degrees of freedom. The known examples of this type, Brink-Schwarz superparticle and Green-Schwarz superstring, have this characteristics [9, 10]. In the present two-dimensional model, there are eight parameters $v_{n}, u_{n \mu}$, $b_{n}, \xi_{n}, \xi_{n \mu}$ and $\tilde{\xi}_{n}$ for each stage of the reducibility. The "real" number of gauge-fixing conditions is $6-2=4$, where six gauge-fixing conditions $\partial^{\mu} C_{n-1 \mu}^{F, B}=0, \epsilon_{\mu \nu}^{-1} \partial^{\nu} \widetilde{C}_{n-1}^{F, B}=0$ are linearly dependent due to $\partial^{\mu}\left(\epsilon_{\mu \nu}^{-1} \partial^{\nu} \widetilde{C}_{n-1}^{F, B}\right)=0$ and thus we needed to impose an extra condition $\partial_{\mu} \bar{C}_{n}^{F, B \mu}=0$.

\section{Quantization in Hamiltonian formulation}

In this section we investigate the quantization of the same model (3.1) in the Hamiltonian formulation and show that the gauge-fixed action obtained from the Hamiltonian formulation coincides with that of the Lagrangian formulation if we make a proper choice of gauge fermion and a suitable identification of ghost fields. One of the important aim of carrying out the quantization of the same model in the Hamiltonian formulation is to see how the regularity violating constraints can be interpreted in the Hamiltonian formulation. In the previous paper on the analysis of the abelian version of the present model, it was pointed out that a physical degree of freedom which does not exist in the classical level appears in the quantum level and the origin of the appearance is essentially related to the violation of regularity [6]. This situation is unchanged in the nonabelian version of the present model and the Hamiltonian formulation of the quantization confirms the result.

\subsection{Purely bosonic model}

For simplicity we first investigate the case where the classical action includes only bosonic fields. Incorporation of fermionic classical fields can be done straightforwardly and will be explained in the next subsection. Following to the standard procedure, we obtain canonical momenta from the action (3.1) where fermionic classical fields are omitted,

$$
\begin{aligned}
\pi_{\phi} & =0, \\
\pi_{\omega_{0}} & =0, \\
\pi_{\omega_{1}} & =-\phi \\
\pi_{B} & =0 .
\end{aligned}
$$


All of these equations give primary constraints. The canonical Hamiltonian following from the Lagrangian becomes

$$
H_{C}=\int d x^{1} \operatorname{Tr}\left\{-\phi D_{1} \omega_{0}+\phi^{2} B\right\}
$$

where $D_{1} \equiv \partial_{1}+\left[\omega_{1},\right]$ is the space component of the covariant derivative. This Hamiltonian and the above primary constraints with Lagrange multipliers define the total Hamiltonian

$$
H_{T}=\int d x^{1} \operatorname{Tr}\left\{-\phi D_{1} \omega_{0}+\phi^{2} B+\lambda_{\phi} \pi_{\phi}+\lambda_{\omega_{0}} \pi_{\omega_{0}}+\lambda_{\omega_{1}}\left(\pi_{\omega_{1}}+\phi\right)+\lambda_{B} \pi_{B}\right\}
$$

According to the ordinary Dirac's procedure, we have to check the consistency of the constraints. The results are

$$
\begin{aligned}
\partial_{0} \pi_{\phi} & =D_{1} \omega_{0}-\{\phi, B\}-\lambda_{\omega_{1}}=0, \\
\partial_{0} \pi_{\omega_{0}} & =-D_{1} \phi=0, \\
\partial_{0}\left(\pi_{\omega_{1}}+\phi\right) & =-\left[\phi, \omega_{0}\right]+\lambda_{\phi}=0, \\
\partial_{0} \pi_{B} & =-\phi^{2}=0 .
\end{aligned}
$$

Two Lagrange multipliers, $\lambda_{\omega_{1}}$ and $\lambda_{\phi}$, are determined from (4.5) and (4.7). After the substitution of these expressions into the total Hamiltonian, we obtain

$$
H_{T}=\int d x^{1} \operatorname{Tr}\left\{-\omega_{0}\left(D_{1} \pi_{\omega_{1}}-\left[\pi_{\phi}, \phi\right]\right)-\left(\phi^{2}+\left\{\pi_{\omega_{1}}, \phi\right\}\right) B+\lambda_{\omega_{0}} \pi_{\omega_{0}}+\lambda_{B} \pi_{B}\right\} .
$$

At the same time, we have found secondary constraints

$$
\begin{aligned}
& D_{1} \phi=0, \\
& -\phi^{2}=0 .
\end{aligned}
$$

The consistency check of these constraints gives no further relations. Thus we have obtained the set of the constraints eqs.(4.1) - (4.4) and eqs.(4.10) and (4.11).

It should be noted that the constraints (4.10) and (4.11) violate Dirac's regularity condition. Constraints are called regular if any function of canonical variables vanishing on the constraint surface can be written as their "linear" combination, where the coefficients of the combination could be dependent on canonical variables. More precisely to say, constraints $\left\{\Phi_{1}, \cdots, \Phi_{M}\right\}$ are regular if independent constraints $\Phi_{1}, \cdots, \Phi_{M^{\prime}}\left(M^{\prime} \leq M\right)$ can be taken as the first $M^{\prime}$ coordinates of the $N$-dimensional phase space in the vicinity of the $\left(N-M^{\prime}\right)$-dimensional constraint surface and thus 
$d \Phi_{1} \wedge \cdots \wedge d \Phi_{M^{\prime}} \neq 0$ on the surface. In the models with nonabelian gauge algebras there are two possibilities depending on the chosen gauge algebra. Firstly, if we take Clifford algebras $c(k, 0)$ or $c(0, k)$ where the metric of the algebra is positive definite or negative definite, then the equation $\phi^{2}=0$ is equivalent to $\phi=0$ which should be the constraint surface determined by (4.10) and (4.11). Neither (4.10) nor (4.11) can be taken as the above constraint for $M^{\prime}=1$ because eq. (4.10) does not imply $\phi=0$ while $\phi^{2}=0$ is not regular since $d \phi^{2}=0$ at $\phi=0$. In this case we can replace these two constraints by a single equivalent constraint

$$
\phi=0
$$

Then we can separate the constraints into the first class and the second class. With some redefinitions we can obtain a set of constraints as

$$
\begin{aligned}
\text { second class } & \pi_{\phi}=0, \quad \phi=0, \\
\text { first class } & \pi_{\omega_{0}}=0, \\
& \pi_{\omega_{1}}=0, \\
& \pi_{B}=0 .
\end{aligned}
$$

These constraints imply that there exist no dynamical variables, which is expected from the topological nature of the generalized Chern-Simons action. The quantization of this system is trivial for the flat base manifold since there is no dynamical degrees of freedom. Finite degrees of freedom may appear depending on the choice of a nontrivial topology for the base manifold. By adopting gauge-fixing conditions $\omega_{0}=\omega_{1}=B=0$, all variables and thus Hamiltonian $H_{T}$ itself vanishes identically. Thus we can conclude this theory is completely empty. However this treatment does not lead to the quantized Lagrangian which we have obtained in the Lagrangian formulation.

The other situation is the case where $\phi^{2}=0$ is not equivalent to $\phi=0$. The specific examples of such algebras are Clifford algebras $c(m, n)(m \neq 0, n \neq 0)$. In particular we now consider $c(2,1)$ algebra generated by $\left\{T_{a}\right\}=\left\{1, \sigma_{1}, \sigma_{2}, i \sigma_{3}\right\}$ where $\sigma_{k}$ 's are Pauli matrices. Then $\phi$ is expanded into components as $\phi=\phi^{s} 1+\phi^{1} \sigma_{1}+\phi^{2} \sigma_{2}+\phi^{3} i \sigma_{3}$, and thus $\phi^{2}=0$ leads to

$$
\left(\phi^{s}\right)^{2}+\left(\phi^{1}\right)^{2}+\left(\phi^{2}\right)^{2}-\left(\phi^{3}\right)^{2}=0, \quad \phi^{s} \phi^{k}=0
$$

These equations are equivalent to

$$
\phi^{s}=0,
$$




$$
\left(\phi^{1}\right)^{2}+\left(\phi^{2}\right)^{2}-\left(\phi^{3}\right)^{2}=0
$$

Eq. (4.13) implies that the constraint surface is not a single but a branched hypersurface in the phase space since $\phi^{3}= \pm \sqrt{\left(\phi^{1}\right)^{2}+\left(\phi^{2}\right)^{2}}$. In the case of $c(k, 0)$ and $c(0, k)$ single regular constraint $\phi=0$ can replace the constraints (4.10) and (4.11) while one of the branch of $\phi^{3}= \pm \sqrt{\left(\phi^{1}\right)^{2}+\left(\phi^{2}\right)^{2}}$ cannot be taken as a regular constraint in the present case since one of the branced surface is not enough to specify whole the constraint surface and furthermore they themselves are singular at $\phi=0$. Therefore it seems rather natural in the generalized Chern-Simons theory to adopt a quantization method different from the usual one, i.e., a quantization based on regularity violating constraints that follow directly from the Lagrangian. In the following we perform the Hamiltonian BRST quantization à la Batalin, Fradkin and Vilkovisky by using the regularity violating constraints. It is interesting that in this treatment of Hamiltonian formulation with a suitable choice of gauge-fixing conditions, we can show that the gauge-fixed action obtained from the regularity violating constraint is just the same as the result of the Lagrangian formulation. Though the Lagrangian and Hamiltonian constructions are formally equivalent in usual cases as shown in refs. [7] and [8], we can show the equivalence of two formulations in the present model only if we adopt the regularity violating constraints in the Hamiltonian formulation.

We first rearrange the constraints $(4.1)-(4.4),(4.10)$ and 4.11 into (4.1) - 4.4 and

$$
\begin{array}{r}
-D_{1} \pi_{\omega_{1}}-\left[\pi_{\phi}, \pi_{\omega_{1}}\right]=0 \\
-\pi_{\omega_{1}}^{2}=0
\end{array}
$$

so that first class constraints, (4.2), (4.4), (4.14) and (4.15), and second class constraints, (4.1) and (4.3), are separated. We can now carry on without the variables $\phi$ and $\pi_{\phi}$ because of the second class constraints, that is, we can replace all $\phi$ by $-\pi_{\omega_{1}}$ and set $\pi_{\phi}$ to be zero by using Dirac's brackets. We further adopt gauge conditions $\omega_{0}=B=0$ for the first class constraints (4.2) and (4.4), for simplicity. Then we can also eliminate $\omega_{0}, \pi_{\omega_{0}}, B$ and $\pi_{B}$ from the system. After these manipulations, we have two phase space variables $\omega_{1}$ and $\pi_{\omega_{1}}$ with the first class constraints

$$
\begin{aligned}
& G_{1} \equiv-D_{1} \pi_{\omega_{1}}=0 \\
& H_{1} \equiv-\pi_{\omega_{1}}^{2}=0
\end{aligned}
$$


and the total Hamiltonian (4.9) vanishes completely.

This system with the first class constraints (4.16) and (4.17) violating the regularity condition is infinitely reducible, as it has been in the Lagrangian formulation:

$$
\begin{aligned}
G_{n} & \equiv(-)^{n-1} D_{1} H_{n-1}+\left[\pi_{\omega_{1}}, G_{n-1}\right]_{(-)^{n}} \\
& =(-)^{n-1}\left[D_{1} \pi_{\omega_{1}}, H_{n-2}\right]_{(-)^{n}}+\left[\pi_{\omega_{1}}^{2}, G_{n-2}\right] \\
& =0, \\
H_{n} & \equiv\left[\pi_{\omega_{1}}, H_{n-1}\right]_{(-)^{n-1}} \\
& =\left[\pi_{\omega_{1}}^{2}, H_{n-2}\right] \\
& =0, \quad n=2,3,4, \cdots .
\end{aligned}
$$

where we have used the constraints (4.16) and (4.17). In the case for $n=2$ the relations are satisfied trivially.

Here we comment on the number of linearly independent constraints. If there are first class constraints the number of the physical degrees of freedom is reduced by twice the number of the first class constraints, since the constraint itself kills one degree and the corresponding gauge symmetry induces another unphysical degree. In the counting of degrees of freedom, the multiplication of the gauge degrees of freedom by the dimension of the gauge algebra should be understood and will be omitted from the discussions. In the present model we have two phase space variables $\omega_{1}$ and $\pi_{\omega_{1}}$. These degrees of freedom should be cancelled by one first class constraint so that the theory is topological and thus has no degrees of freedom even after the quantization. Since we have two first class constraints (4.16) and (4.17), there should be one relation between them, in other words (4.16) and (4.17) are linearly dependent. In fact what happened in the present model is that two reducibility conditions appear at each level. According to the above argument the number of linearly independent equations for (4.18) and (4.19) at each level should also be one. In order to compensate the over cancelled degrees of freedom, two reducibility conditions appear again and then the process repeats infinite times. This is how the infinite reducibility appears in the Hamiltonian formulation.

In the case of a simple constrained system, we can quantize the system without unphysical degrees of freedom by solving the constraints. In many cases, however, we lose manifest invariance under important global symmetries and/or the locality. Furthermore in the present case the solutions of the constraints (4.16) and (4.17) are 
determined according to the gauge algebra, as explained above. We will show that the action obtained in the Hamiltonian formulation coincides with the result of the Lagrangian formulation which contains infinitely many unphysical degrees of freedom, i.e., ghost fields. We adopt the Hamiltonian formulation given by Batalin, Fradkin and Vilkovisky which accommodates the reducibility of the system. In this formulation a phase space is extended so as to contain ghosts and ghost momenta. Then a nilpotent BRST differential is constructed and a physical phase space is defined as its cohomology which is a set of gauge invariant functions on the constraint surface. The role of the ghost momenta is to exclude functions vanishing on the constraint surface from the cohomology and gauge variant functions are removed from the cohomology because of the action of the BRST differential for the ghosts.

First we introduce infinite ghosts and ghost momenta

$$
\begin{array}{llll}
\eta_{2 n}^{B}, & \eta_{2 n}^{\prime B}, & P_{2 n}^{B}, & P_{2 n}^{\prime B}, \\
\eta_{2 n-1}^{F}, & \eta_{2 n-1}^{\prime F}, & P_{2 n-1}^{F}, & P_{2 n-1}^{\prime F},
\end{array} \quad n=1,2,3, \cdots,
$$

where $\eta_{n}^{B, F}$ and $P_{n}^{B, F}$ correspond with the constraint (4.16) and the reducibility (4.18) and $\eta_{n}^{\prime B, F}$ and $P_{n}^{\prime B, F}$ with (4.17) and (4.19). The ghost numbers of the $\eta_{n}^{B, F}$ and $\eta_{n}^{\prime B, F}$ are $n$ and those of $P_{n}^{B, F}$ and $P_{n}^{\prime B, F}$ are $-n$. The fields with index $B$ and $F$ are bosonic and fermionic, respectively. Although the way for constructing the BRST differential in the Hamiltonian formulation is systematic, it is complicated in the present case due to the infinite reducibility. After the step by step construction according to the systematic procedure, we have found an elegant way of presenting the result. Thus we first give the result we obtained and then show that it is just what the usual procedure leads to. The construction which we carried out is similar to that in the Lagrangian formulation. First we define a generalized gauge field $\widetilde{\mathcal{A}}$ from the ghosts, $\eta_{n}^{B, F}$ and $\eta_{n}^{\prime B, F}$, and the ghost momenta, $P_{n}^{B, F}$ and $P_{n}^{\prime B, F}$, by

$$
\begin{gathered}
\widetilde{\mathcal{A}}=\mathbf{1} \psi+\mathbf{i} \hat{\psi}+\mathbf{j} A+\mathbf{k} \hat{A} \\
\psi=\sum_{n=-\infty}^{\infty} \Gamma_{2 n-1}^{F} d x^{1} \equiv \sum_{n=1}^{\infty}\left(\eta_{2 n-1}^{\prime F}+P_{2 n-1}^{F}\right) d x^{1}, \\
\hat{\psi}=\sum_{n=-\infty}^{\infty} \Pi_{2 n-1}^{F} \equiv \sum_{n=1}^{\infty}\left(\eta_{2 n-1}^{F}+P_{2 n-1}^{\prime F}\right), \\
A=\sum_{n=-\infty}^{\infty} \Gamma_{2 n}^{B} d x^{1} \equiv\left\{\omega_{1}+\sum_{n=1}^{\infty}\left(\eta_{2 n}^{\prime B}-P_{2 n}^{B}\right)\right\} d x^{1}, \\
\hat{A}=\sum_{n=-\infty}^{\infty} \Pi_{2 n}^{B} \equiv \pi_{\omega_{1}}+\sum_{n=1}^{\infty}\left(\eta_{2 n}^{B}+P_{2 n}^{\prime B}\right) .
\end{gathered}
$$


We next introduce the BRST differential $s$ as

$$
s \widetilde{\mathcal{A}}=-\widetilde{\mathcal{F}} \mathbf{i}=-\left(Q^{\prime} \widetilde{\mathcal{A}}+\widetilde{\mathcal{A}}^{2}\right) \mathbf{i}
$$

where $Q^{\prime} \equiv \mathbf{j} d x^{1} \partial_{1}$ is one dimensional exterior derivative which does not include the time derivative. It should be noted that the BRST differential has the same form as the Lagrangian counterpart. The nilpotency of the BRST differential, $s^{2}=0$, can be shown in the same way as in the Lagrangian formulation. The explicit actions of the BRST differential are

$$
\begin{aligned}
s \Pi_{2 n}^{B} & =\sum_{m=-\infty}^{\infty}\left[\Pi_{2 m}^{B}, \Pi_{2(n-m)+1}^{F}\right] \\
s \Pi_{2 n-1}^{F} & =\sum_{m=-\infty}^{\infty}\left(\frac{1}{2}\left\{\Pi_{2 m}^{B}, \Pi_{2(n-m)}^{B}\right\}+\frac{1}{2}\left\{\Pi_{2 m-1}^{F}, \Pi_{2(n-m)+1}^{F}\right\}\right) \\
s \Gamma_{2 n}^{B} & =\partial_{1} \Pi_{2 n+1}^{F}+\sum_{m=-\infty}^{\infty}\left(\left[\Gamma_{2 m}^{B}, \Pi_{2(n-m)+1}^{F}\right]-\left\{\Pi_{2 m}^{B}, \Gamma_{2(n-m)+1}^{F}\right\}\right), \\
s \Gamma_{2 n-1}^{F} & =\partial_{1} \Pi_{2 n}^{B}+\sum_{m=-\infty}^{\infty}\left(\left[\Gamma_{2 m}^{B}, \Pi_{2(n-m)}^{B}\right]+\left\{\Gamma_{2 m-1}^{F}, \Pi_{2(n-m)+1}^{F}\right\}\right) .
\end{aligned}
$$

We now show that $s$ coincides with the BRST differential which is obtained by the formulation of Batalin, Fradkin and Vilkovisky. In order to obtain the BRST differential we follow to the systematic procedure developed by Henneaux et al. [5]. The BRST differential can be decomposed into $s=\delta+D+\sum_{k \geq 1} \stackrel{(k)}{s}$. The homology of the Koszul-Tate differential $\delta$ is a set of functions on the constraint surface. The extended longitudinal differential $D$ is considered on the homology of the Koszul-Tate differential and its cohomology is a set of gauge invariant functions. Finally $\stackrel{(k)}{s}$ is determined so that $s$ is nilpotent. Then it is guaranteed by the (co)homological perturbation theory that the cohomology of the BRST differential is a set of gauge invariant functions on the constraint surface.

We first define the antighost number as

$$
\begin{aligned}
\operatorname{antigh}(\delta) & =-1, \quad \operatorname{antigh}(D)=0, \quad \operatorname{antigh}(\stackrel{(k)}{s})=k, \\
\operatorname{antigh}\left(\omega_{1}\right) & =\operatorname{antigh}\left(\pi_{\omega_{1}}\right)=0, \\
\operatorname{antigh}\left(P_{2 n}^{B}\right) & =\operatorname{antigh}\left(P_{2 n}^{\prime B}\right)=2 n, \\
\operatorname{antigh}\left(P_{2 n-1}^{F}\right) & =\operatorname{antigh}\left(P_{2 n-1}^{\prime F}\right)=2 n-1 .
\end{aligned}
$$

The actions of the Koszul-Tate differential $\delta$ for the ghost momenta are read from 
eqs.(4.23) by comparing antighost numbers:

$$
\begin{aligned}
\delta P_{2 n+1}^{F}= & D_{1} P_{2 n}^{\prime B}+\left[\pi_{\omega_{1}}, P_{2 n}^{B}\right] \\
& -\sum_{m=1}^{n-1}\left[P_{2 m}^{B}, P_{2(n-m)}^{\prime B}\right]+\sum_{m=0}^{n-1}\left\{P_{2 m+1}^{F}, P_{2(n-m)-1}^{\prime F}\right\}, \\
\delta P_{2 n}^{B}= & -D_{1} P_{2 n-1}^{\prime F}+\left\{\pi_{\omega_{1}}, P_{2 n-1}^{F}\right\} \\
& +\sum_{m=1}^{n-1}\left[P_{2 m}^{B}, P_{2(n-m)-1}^{\prime F}\right]+\sum_{m=1}^{n-1}\left\{P_{2 m}^{\prime B}, P_{2(n-m)-1}^{F}\right\}, \\
\delta P_{2 n+1}^{\prime F}= & \left\{\pi_{\omega_{1}}, P_{2 n}^{\prime B}\right\}+\frac{1}{2} \sum_{m=1}^{n-1}\left\{P_{2 m}^{\prime B}, P_{2(n-m)}^{\prime B}\right\}+\frac{1}{2} \sum_{m=0}^{n-1}\left\{P_{2 m+1}^{\prime F}, P_{2(n-m)-1}^{\prime} F\right. \\
\delta P_{2 n}^{\prime B}= & {\left[\pi_{\omega_{1}}, P_{2 n-1}^{\prime} F\right]+\sum_{m=1}^{n-1}\left[P_{2 m}^{\prime} B, P_{2(n-m)-1}^{\prime} F\right.}
\end{aligned}
$$

in particular

$$
\begin{gathered}
\delta P_{1}^{F}=D_{1} \pi_{\omega_{1}}, \\
\delta P_{1}^{\prime F}=\pi_{\omega_{1}}^{2} .
\end{gathered}
$$

It is understood that these coincide with the actions of the Koszul-Tate differential constructed from the constraints (4.16) and (4.17) and the reducibilities (4.18) and (4.19).

We next consider the extended longitudinal differential $D$. In cases with reducibilities, it is expanded into $D=\Delta+d+\sum_{k \leq-1} \stackrel{(k)}{D}$ where $d$ is called the longitudinal differential and $\Delta$ the auxiliary differential and the auxiliary degree is assigned as

$$
\begin{aligned}
\operatorname{aux}(\Delta) & =1, \quad \operatorname{aux}(d)=0, \quad \operatorname{aux}(\stackrel{(k)}{D})=k, \\
\operatorname{aux}\left(\omega_{1}, \pi_{\omega_{1}}\right) & =0=\operatorname{aux}\left(\eta_{1}^{F}, \eta_{1}^{\prime F}\right), \\
\operatorname{aux}\left(\eta_{2 n}^{B}, \eta_{2 n}^{B}\right) & =2 n-1, \\
\operatorname{aux}\left(\eta_{2 n+1}^{F}, \eta_{2 n+1}^{\prime F}\right) & =2 n, \quad n=1,2,3, \cdots .
\end{aligned}
$$

The operations of the longitudinal differential $d$ for $\omega_{1}, \pi_{\omega_{1}}, \eta_{1}^{F}$ and $\eta_{1}^{\prime F}$ are determined from the form of the gauge transformations and the nilpotency of $d$ :

$$
\begin{aligned}
d \omega_{1} & =D_{1} \eta_{1}^{F}-\left\{\pi_{\omega_{1}}, \eta_{1}^{\prime F}\right\}, \\
d \pi_{\omega_{1}} & =\left[\pi_{\omega_{1}}, \eta_{1}^{F}\right] \\
d \eta_{1}^{F} & =\eta_{1}^{F^{2}} \\
d \eta_{1}^{\prime F} & =\left\{\eta_{1}^{\prime F}, \eta_{1}^{F}\right\} .
\end{aligned}
$$


The actions of $\Delta$ are obtained from the reducibilities (4.18) and (4.19) as

$$
\begin{aligned}
\Delta \omega_{1} & =0=\Delta \pi_{\omega_{1}}, \\
\Delta \eta_{2 n}^{B} & =\left[\pi_{\omega_{1}}, \eta_{2 n+1}^{F}\right], \\
\Delta \eta_{2 n-1}^{F} & =\left\{\pi_{\omega_{1}}, \eta_{2 n}^{B}\right\}, \\
\Delta \eta_{2 n}^{\prime B} & =D_{1} \eta_{2 n+1}^{F}-\left\{\pi_{\omega_{1}}, \eta_{2 n+1}^{\prime F}\right\}, \\
\Delta \eta_{2 n-1}^{\prime F} & =D_{1} \eta_{2 n}^{B}-\left[\pi_{\omega_{1}}, \eta_{2 n}^{\prime B}\right] .
\end{aligned}
$$

The transformation coefficients of the auxiliary differential $\Delta$ with respect to ghosts correspond to the transpose of those of the Koszul-Tate differential $\delta$ except for the bilinear terms of the ghost momenta in eqs. (4.24) [5]. The transformation property of the longitudinal differential $d$ for the higher ghost number ghost fields can be obtained by imposing $(d \Delta+\Delta d) \eta_{n}=0$ and $(d \Delta+\Delta d) \eta_{n}^{\prime}=0$ iteratively. To be more specific we use eqs.(4.25) and (4.26) as starting relations, then $D$ is determined order by order of the auxiliary degree by requiring the nilpotency of $D$ on the constraint surface. The results coincide with those obtained from eqs.(4.23):

$$
\begin{aligned}
D \omega_{1}= & d \omega_{1}=D_{1} \eta_{1}^{F}-\left\{\pi_{\omega_{1}}, \eta_{1}^{\prime F}\right\} \\
D \pi_{\omega_{1}}= & d \pi_{\omega_{1}}=\left[\pi_{\omega_{1}}, \eta_{1}^{F}\right] \\
D \eta_{2 n}^{B}= & {\left[\pi_{\omega_{1}}, \eta_{2 n+1}^{F}\right]+\sum_{m=1}^{n}\left[\eta_{2 m}^{B}, \eta_{2(n-m)+1}^{F}\right] } \\
D \eta_{2 n-1}^{F}= & \left\{\pi_{\omega_{1}}, \eta_{2 n}^{B}\right\}+\frac{1}{2} \sum_{m=1}^{n-1}\left\{\eta_{2 m}^{B}, \eta_{2(n-m)}^{B}\right\}+\frac{1}{2} \sum_{m=0}^{n-1}\left\{\eta_{2 m+1}^{F}, \eta_{2(n-m)-1}^{F}\right\} \\
D \eta_{2 n}^{\prime B}= & D_{1} \eta_{2 n+1}^{F}-\left\{\pi_{\omega_{1}}, \eta_{2 n+1}^{\prime F}\right\} \\
& +\sum_{m=1}^{n}\left(\left[\eta_{2 m}^{\prime B}, \eta_{2(n-m)+1}^{F}\right]-\left\{\eta_{2 m}^{B}, \eta_{2(n-m)+1}^{\prime F}\right\}\right) \\
D \eta_{2 n-1}^{\prime F}= & D_{1} \eta_{2 n}^{B}-\left[\pi_{\omega_{1}}, \eta_{2 n}^{\prime B}\right]+\sum_{m=1}^{n-1}\left[\eta_{2 m}^{\prime B}, \eta_{2(n-m)}^{B}\right]+\sum_{m=0}^{n-1}\left\{\eta_{2 m+1}^{\prime F}, \eta_{2(n-m)-1}^{F}\right\}
\end{aligned}
$$

Then the actions of $D$ for the ghost momenta and of $\stackrel{(k)}{s}$ for all fields are determined order by order of the antighost number by requiring the nilpotency of the BRST differential $s$. It is convincing that the result obtained by these procedures leads to the BRST differential (4.22) which is nilpotent by its construction. For completeness we give the action of $D$ for the ghost momenta and of $\stackrel{(k)}{s}$ for all fields in the appendix.

The extended phase space is defined to include the ghosts and ghost momenta with a canonical structure

$$
\left[\pi_{\omega_{1}}, \omega_{1}\right]_{P}=-1
$$




$$
\begin{aligned}
& {\left[P_{2 m}^{B}, \eta_{2 n}^{B}\right]_{P}=\left[P_{2 m-1}^{F}, \eta_{2 n-1}^{F}\right]_{P}=-\delta_{m n},} \\
& {\left[P_{2 m}^{\prime B}, \eta_{2 n}^{\prime B}\right]_{P}=\left[P_{2 m-1}^{\prime F}, \eta_{2 n-1}^{\prime F}\right]_{P}=-\delta_{m n},}
\end{aligned}
$$

where $[,]_{P}$ represents the graded Poisson bracket which will be replaced by the graded commutation relation $\left([A, B]_{P} \rightarrow A B-(-)^{|A||B|} B A\right.$, with $|A|,|B|$ Grassmann parity of the fields $A$ and $B$ ) multiplied by $-i$ upon the quantization, as usual. Here we have not explicitly shown the coordinate dependence. For example the first relation in the above actually means $\left.\left[\pi_{\omega_{1}}\left(x_{0}, x_{1}\right), \omega_{1}\left(y_{0}, y_{1}\right)\right]_{P}\right|_{x_{0}=y_{0}}=-\delta\left(x_{1}-y_{1}\right)$. Hereafter we omit the coordinate dependence in the Poisson bracket relation. These equations can be written in the compact forms

$$
\left[\Pi_{2 m}^{B}, \Gamma_{2 n}^{B}\right]_{P}=\left[\Pi_{2 m-1}^{F}, \Gamma_{2 n+1}^{F}\right]_{P}=-\delta_{m+n, 0}
$$

By using this canonical relation, the nilpotent BRST charge $\Omega_{m i n}$, which realizes $s X=$ $\left[X, \Omega_{m i n}\right]_{P}$ for $X=\Pi_{2 m}^{B}, \Pi_{2 m-1}^{F}, \Gamma_{2 n}^{B}$ and $\Gamma_{2 n+1}^{F}$, is defined by

$$
\begin{aligned}
\Omega_{\text {min }}= & \int \operatorname{Tr}_{\mathbf{1}}^{1}\left(\frac{1}{2} \widetilde{\mathcal{A}} Q^{\prime} \widetilde{\mathcal{A}}+\frac{1}{3} \widetilde{\mathcal{A}}^{3}\right) \\
= & \int \operatorname{Tr}^{1}\left\{\hat{A}\left(d_{1} \hat{\psi}+[A, \hat{\psi}]\right)-\psi\left(d_{1} A+A^{2}+\hat{\psi}^{2}+\hat{A}^{2}\right)+\frac{1}{3} \psi^{3}\right\} \\
= & \int d x^{1} \operatorname{Tr} \sum_{n=-\infty}^{\infty}\left\{\Pi_{2 n}^{B}\left(\partial_{1} \Pi_{-2 n+1}^{F}+\sum_{m=-\infty}^{\infty}\left[\Gamma_{2 m}^{B}, \Pi_{-2(m+n)+1}^{F}\right]\right)\right. \\
& \left.\quad-\Gamma_{2 n+1}^{F} \sum_{m=-\infty}^{\infty}\left(\Pi_{2 m-1}^{F} \Pi_{-2(m+n)+1}^{F}+\Pi_{2 m}^{B} \Pi_{-2(m+n)}^{B}\right)\right\},
\end{aligned}
$$

where the integration is performed on the one-dimensional space and $d_{1}=d x^{1} \partial_{1}$. In one dimensional expression in the above, the terms $d_{1} A, A^{2}, \psi^{3}$ vanish. The upper index 1 on the Tr indicates to pick up only the part with ghost number 1 and the subscript 1 in eq. (4.30) is necessary in order that the integrant contains only fermionic odd forms. It is very interesting to note that the BRST charge $\Omega_{\min }$ in the minimal sector is the fermionic counterpart of the same generalized Chern-Simons action in one dimension, as we can compare the above expression with $S_{o}^{f}$ in (2.10).

In order to fix the gauge, we have to extend the phase space further. Since we want to make the Landau type gauge-fixing as in the Lagrangian formulation we introduce the following set of canonical variables and momenta:

$$
\begin{aligned}
& \lambda_{2(n-1)}^{B}, \quad \lambda_{2 n-1}^{F}, \quad b_{2(n-1)}^{B}, \quad b_{2 n-1}^{F}, \quad \bar{C}_{2 n}^{B}, \quad \bar{C}_{2 n-1}^{F}, \quad \rho_{2 n}^{B}, \quad \rho_{2 n-1}^{F}, \\
& \lambda_{2(n-1)}^{\prime B}, \quad \lambda_{2 n-1}^{\prime F}, \quad b_{2(n-1)}^{\prime B}, \quad b_{2 n-1}^{\prime F}, \quad \bar{C}_{2 n}^{\prime B}, \quad \bar{C}_{2 n-1}^{\prime F}, \quad \rho_{2 n}^{\prime B}, \quad \rho_{2 n-1}^{\prime F}, \\
& \lambda_{2(n-1)}^{\prime \prime B}, \quad \lambda_{2 n-1}^{\prime \prime F}, \quad b_{2(n-1)}^{\prime \prime B}, \quad b_{2 n-1}^{\prime \prime F}, \quad \bar{C}_{2 n}^{\prime \prime B}, \quad \bar{C}_{2 n-1}^{\prime \prime} F, \quad \rho_{2 n}^{\prime \prime B}, \quad \rho_{2 n-1}^{\prime \prime F}, \quad n=1,2,3, \cdots,
\end{aligned}
$$


where the ghost number of $\lambda_{n}$ and $\rho_{n}$ is $n$ while that of $\bar{C}_{n}$ and $b_{n}$ is $-n$. The statistics of the even (odd) ghost number fields is bosonic (fermionic). The canonical structure is defined by

$$
\begin{aligned}
{\left[\rho_{2 m}^{B}, \bar{C}_{2 n}^{B}\right]_{P}=-\delta_{m n}, } & {\left[b_{2 m}^{B}, \lambda_{2 n}^{B}\right]_{P}=-\delta_{m n}, } \\
{\left[\rho_{2 m-1}^{F}, \bar{C}_{2 n-1}^{F}\right]_{P}=-\delta_{m n}, } & {\left[b_{2 m-1}^{F}, \lambda_{2 n-1}^{F}\right]_{P}=-\delta_{m n} }
\end{aligned}
$$

and the similar canonical structure is defined for the primed fields. The action of the BRST differential is also extended to these variables as

$$
\begin{aligned}
& s \lambda_{2 n}^{B}=\rho_{2 n+1}^{F}, \quad s \rho_{2 n+1}^{F}=0, \quad s \bar{C}_{2 n-1}^{F}=-b_{2 n}^{B}, \quad s b_{2 n}^{B}=0, \\
& s \lambda_{2 n-1}^{F}=\rho_{2 n}^{B}, \quad s \rho_{2 n}^{B}=0, \quad s \bar{C}_{2 n}^{B}=-b_{2 n+1}^{F}, \quad s b_{2 n+1}^{F}=0,
\end{aligned}
$$

and those for the primed fields are defined in the same way. The corresponding extended BRST charge is given by

$$
\begin{aligned}
& \Omega=\Omega_{\text {min }}+\Omega_{\text {nonmin }}, \\
& \Omega_{\text {nonmin }}=\int d x^{1} \operatorname{Tr} \sum_{n=1}^{\infty}\left(b_{2(n-1)}^{B} \rho_{2 n-1}^{F}+b_{2(n-1)}^{\prime B} \rho_{2 n-1}^{\prime F}+b_{2(n-1)}^{\prime \prime B} \rho_{2 n-1}^{\prime \prime F}\right. \\
&\left.-b_{2 n-1}^{F} \rho_{2 n}^{B}-b_{2 n-1}^{\prime F} \rho_{2 n}^{\prime B}-b_{2 n-1}^{\prime \prime F} \rho_{2 n}^{\prime \prime B}\right) .
\end{aligned}
$$

Now the gauge-fixed action $S$ is obtained by a Legendre transformation from the Hamiltonian in the extended phase space:

$$
\begin{aligned}
& S=\int d x^{0}\left\{\int d x ^ { 1 } \operatorname { T r } \left(\dot{\omega}_{1} \pi_{\omega_{1}}+\sum_{n=1}^{\infty}\left(\dot{\eta}_{2 n}^{B} P_{2 n}^{B}+\dot{\eta}_{2 n-1}^{F} P_{2 n-1}^{F}+\dot{\eta}_{2 n}^{\prime}{ }_{2 n}^{B} P_{2 n}^{\prime B}+\dot{\eta}^{\prime}{ }_{2 n-1}^{F} P_{2 n-1}^{\prime} F\right.\right.\right. \\
& +\dot{\lambda}_{2(n-1)}^{B} b_{2(n-1)}^{B}+\dot{\lambda}_{2 n-1}^{F} b_{2 n-1}^{F}+\dot{\lambda}_{2(n-1)}^{\prime B} b_{2(n-1)}^{\prime B}+\dot{\lambda}_{2 n-1}^{\prime F} b_{2 n-1}^{\prime F} \\
& +\dot{\lambda}_{2(n-1)}^{\prime \prime B} b_{2(n-1)}^{\prime \prime B}+\dot{\lambda}_{2 n-1}^{\prime \prime} F b_{2 n-1}^{\prime \prime F}+\dot{\bar{C}}_{2 n}^{B} \rho_{2 n}^{B}+\dot{\bar{C}}_{2 n-1}^{F} \rho_{2 n-1}^{F} \\
& \left.\left.\left.+\dot{\bar{C}}_{2 n}^{\prime B} \rho_{2 n}^{\prime B}+\dot{\bar{C}}_{2 n-1}^{F} \rho_{2 n-1}^{\prime F}+\dot{\bar{C}}_{2 n}^{\prime \prime}{ }_{2 n}^{B} \rho_{2 n}^{\prime \prime B}+\dot{\bar{C}}_{2 n-1}^{\prime F} \rho_{2 n-1}^{\prime \prime F}\right)\right)-H_{K}\right\}, \\
& H_{K}=[K, \Omega]_{P},
\end{aligned}
$$

where $K$ is called a gauge-fixing fermion. The gauge-fixed Hamiltonian $H_{K}$ consists of gauge-fixing and ghost parts only since the total Hamiltonian of the system have vanished. There is no systematic way to find $K$ so as to yield a covariant expression. Actually we want to show that the action obtained in the Hamiltonian formulation coincides with that in the Lagrangian formulation. We take the following gauge-fixing fermion $K$ based on the experience of the quantization of $g l(1, \mathbf{R})$ model:

$$
K=\int d x^{1} \operatorname{Tr} \sum_{n=1}^{\infty}\left\{\epsilon_{1} \bar{C}_{2 n-1}^{F} \partial^{1} \eta_{2(n-1)}^{\prime B}+\epsilon_{2} \bar{C}_{2 n}^{B} \partial^{1} \eta_{2 n-1}^{\prime F}+\epsilon_{3} \bar{C}_{2 n-1}^{\prime F} \partial_{1} \lambda_{2(n-1)}^{\prime \prime B}\right.
$$




$$
\begin{aligned}
& +\epsilon_{4} \bar{C}_{2 n}^{\prime B} \partial_{1} \lambda_{2 n-1}^{\prime \prime F}+\epsilon_{5} \bar{C}_{2 n-1}^{\prime \prime F} \partial^{1} \lambda_{2(n-1)}^{\prime B}+\epsilon_{6} \bar{C}_{2 n}^{\prime \prime B} \partial^{1} \lambda_{2 n-1}^{\prime F} \\
& \left.+\epsilon_{7} P_{2 n-1}^{F} \lambda_{2(n-1)}^{B}+\epsilon_{8} P_{2 n}^{B} \lambda_{2 n-1}^{F}+\epsilon_{9} P_{2 n-1}^{\prime F} \lambda_{2(n-1)}^{\prime B}+\epsilon_{10} P_{2 n}^{\prime B} \lambda_{2 n-1}^{\prime F}\right\},
\end{aligned}
$$

where we denote $\omega_{1}$ by $\eta_{0}^{\prime}$ and the sign factors $\epsilon_{i}= \pm 1(i=1,2, \cdots, 10)$ will be determined soon.

We first impose one gauge-fixing condition, which is originated from the terms $\bar{C}_{n}^{B, F} \partial^{1} \eta_{n-1}^{\prime F, B}$ in $K$, at each level of the ghost number in the minimal sector. It is consistent with the fact that the number of the linearly independent constraints or reducibility conditions at each level should be one, as mentioned above. Next we substitute this gauge-fixing fermion into eqs.4.35) and (4.36) and integrate out the momentum variables $P_{n}^{B, F}, P_{n}^{\prime B, F}, \rho_{n}^{B, F}$ and $\rho_{n}^{B, F}$. If we set

$$
\epsilon_{2}=\epsilon_{3}=\epsilon_{7}=\epsilon_{8}=1, \quad \epsilon_{1}=\epsilon_{5}=-1, \quad \epsilon_{6}=-\epsilon_{4},
$$

and rename the variables as

$$
\begin{aligned}
\pi_{\omega_{1}} & \longrightarrow-\phi, \\
\left(\eta_{2 n}^{B}, \eta_{2 n+1}^{F}, \eta_{2 n}^{\prime B}, \eta_{2 n+1}^{\prime F}\right) & \longrightarrow\left(-C_{2 n}^{B}, C_{2 n+1}^{F}, C_{2 n \mu=1}^{B},-C_{2 n-1 \mu=1}^{F}\right), \\
\left(\lambda_{2 n}^{B}, \lambda_{2 n+1}^{F}\right) & \longrightarrow\left(-C_{2 n \mu=0}^{B},-C_{2 n-1 \mu=0}^{F}\right), \\
\left(\lambda_{2 n}^{\prime B}, \lambda_{2 n+1}^{\prime F}\right) & \longrightarrow\left(\epsilon_{9} \widetilde{C}_{2 n}^{B}, \epsilon_{10} \widetilde{C}_{2 n+1}^{F}\right), \\
\left(\lambda_{2 n}^{\prime \prime B}, \lambda_{2 n+1}^{\prime \prime F}\right) & \longrightarrow\left(\epsilon_{9} \eta_{2 n}^{B}, \epsilon_{4} \epsilon_{10} \eta_{2 n+1}^{F}\right), \\
\left(b_{2 n}^{B}, b_{2 n+1}^{F}\right) & \longrightarrow\left(-b_{2 n}^{B},-b_{2 n+1}^{F}\right), \\
\left(b_{2 n}^{\prime B}, b_{2 n+1}^{\prime F}\right) & \longrightarrow\left(\epsilon_{9} b_{2 n}^{B \mu=1}, \epsilon_{10} b_{2 n+1}^{F \mu=1}\right), \\
\left(b_{2 n}^{\prime \prime B}, b_{2 n+1}^{\prime \prime F}\right) & \longrightarrow\left(\epsilon_{9} b_{2 n}^{B \mu=0}, \epsilon_{4} \epsilon_{10} b_{2 n+1}^{F \mu}\right), \\
\left(\bar{C}_{2 n}^{\prime B}, \bar{C}_{2 n+1}^{\prime F}\right) & \longrightarrow\left(-\epsilon_{10} \bar{C}_{2 n}^{B \mu=1},-\epsilon_{9} \bar{C}_{2 n+1}^{F \mu=1}\right), \\
\left(\bar{C}_{2 n}^{\prime \prime B}, \bar{C}_{2 n+1}^{\prime \prime F}\right) & \longrightarrow\left(-\epsilon_{4} \epsilon_{10} \bar{C}_{2 n}^{B \mu=0},-\epsilon_{9} \bar{C}_{2 n+1}^{F \mu=0}\right), \\
\left(\rho_{2 n}^{\prime \prime B}, \quad \rho_{2 n+1}^{\prime \prime F}\right) & \longrightarrow\left(\epsilon_{4} \epsilon_{10} \pi_{2 n}^{B}, \epsilon_{9} \pi_{2 n+1}^{F}\right), \\
C_{n=0}^{B}\left({ }_{n}=0\right. & \widetilde{C}_{0}^{B}=B,
\end{aligned}
$$

this action completely coincides with the gauge-fixed action (3.32) in the Lagrangian formulation in which the propagators of all fields are well-defined evidence to the statement that the number of linearly independent constraints between (4.16) and (4.17) should be one.

* Three sign factors $\epsilon_{4}, \epsilon_{9}, \epsilon_{10}$ have remained arbitrary. 


\subsection{Inclusion of fermions in the Hamiltonian formulation}

In the case where the classical action contains fermionic fields in addition to bosonic fields we can follow the similar procedure as the one carried out in the previous subsection. We explain how the analyses given in the purely bosonic model must be modified.

First we obtain the constraints

$$
\begin{aligned}
\text { second class } & \pi_{\phi}=0, \quad \pi_{\omega_{1}}+\phi=0 \\
& \pi_{\chi}=0, \quad \pi_{\chi_{1}}+\chi=0 \\
\text { first class } & \pi_{\omega_{0}}=0, \quad \pi_{B}=0 \\
& \pi_{\chi_{0}}=0, \quad \pi_{\tilde{\chi}}=0 \\
& D_{1} \pi_{\omega_{1}}+\left[\pi_{\phi}, \pi_{\omega_{1}}\right]+\left\{\pi_{\chi_{1}}, \chi_{1}-\pi_{\chi}\right\}=0 \\
& \pi_{\omega_{1}}^{2}+\pi_{\chi_{1}}^{2}=0 \\
& D_{1} \pi_{\chi_{1}}+\left[\pi_{\phi}, \pi_{\chi_{1}}\right]-\left\{\pi_{\omega_{1}}, \chi_{1}-\pi_{\chi}\right\}=0 \\
& {\left[\pi_{\omega_{1}}, \pi_{\chi_{1}}\right]=0 }
\end{aligned}
$$

and the total Hamiltonian

$$
\begin{aligned}
H_{T}=\int d x^{1} \operatorname{Tr} & \left\{\omega_{0}\left(-D_{1} \pi_{\omega_{1}}-\left[\pi, \pi_{\phi}\right]-\left\{\chi, \pi_{\chi}\right\}-\left\{\chi_{1}, \pi_{\chi_{1}}\right\}\right)\right. \\
& +B\left(-\phi^{2}-\chi^{2}-\left\{\pi_{\omega_{1}}, \phi\right\}-\left\{\chi, \pi_{\chi_{1}}\right\}\right) \\
& +\chi_{0}\left(-D_{1} \pi_{\chi_{1}}+\left[\pi_{\phi}, \chi\right]+\left\{\pi_{\omega_{1}}, \chi_{1}\right\}+\left\{\phi, \pi_{\chi}\right\}\right) \\
& +\tilde{\chi}\left([\phi, \chi]+\left[\pi_{\phi}, \chi\right]+\left\{\pi_{\omega_{1}}, \chi_{1}\right\}+\left\{\phi, \pi_{\chi}\right\}\right) \\
& \left.+\lambda_{\omega_{0}} \pi_{\omega_{0}}+\lambda_{B} \pi_{B}+\lambda_{\chi_{0}} \pi_{\chi_{0}}+\lambda_{\tilde{\chi}} \pi_{\tilde{\chi}}\right\} .
\end{aligned}
$$

Then by using Dirac's brackets for the second class constraints (4.38) and (4.39) and adopting gauge conditions $\omega_{0}=B=\chi_{0}=\tilde{\chi}=0$ for the first class constraints (4.40) and (4.41) we have four phase space variables $\omega_{1}, \pi_{\omega_{1}}, \chi_{1}$ and $\pi_{\chi_{1}}$ with the first class constraints

$$
\begin{aligned}
& G_{1}^{B} \equiv-D_{1} \pi_{\omega_{1}}-\left\{\pi_{\chi_{1}}, \chi_{1}\right\}=0 \\
& H_{1}^{B} \equiv-\pi_{\omega_{1}}^{2}-\pi_{\chi_{1}}^{2}=0 \\
& G_{1}^{F} \equiv D_{1} \pi_{\chi_{1}}-\left\{\pi_{\omega_{1}}, \chi_{1}\right\}=0 \\
& H_{1}^{F} \equiv-\left[\pi_{\omega_{1}}, \pi_{\chi_{1}}\right]=0
\end{aligned}
$$

and now the total Hamiltonian vanishes. It is understood that these constraints are 
infinitely reducible due to the following relations which hold on the constraint surface:

$$
\begin{aligned}
G_{n}^{B} \equiv & (-)^{n+1} D_{1} H_{n-1}^{B}+\left[\pi_{\omega_{1}}, G_{n-1}^{B}\right]_{(-)^{n}} \\
& +(-)^{n+1}\left\{\pi_{\chi_{1}}, G_{n-1}^{F}\right\}+\left[\chi_{1}, H_{n-1}^{F}\right]_{(-)^{n+1}}=0, \\
H_{n}^{B} \equiv & {\left[\pi_{\omega_{1}}, H_{n-1}^{B}\right]_{(-)^{n+1}}+(-)^{n+1}\left\{\pi_{\chi_{1}}, H_{n-1}^{F}\right\}=0, } \\
G_{n}^{F} \equiv & (-)^{n} D_{1} H_{n-1}^{F}+(-)^{n}\left[\pi_{\chi_{1}}, G_{n-1}^{B}\right] \\
& +\left[\chi_{1}, H_{n-1}^{B}\right]_{(-)^{n+1}}+\left[\pi_{\omega_{1}}, G_{n-1}^{F}\right]_{(-)^{n+1}}=0, \\
H_{n}^{F} \equiv & (-)^{n}\left[\pi_{\chi_{1}}, H_{n-1}^{B}\right]+\left[\pi_{\omega_{1}}, H_{n-1}^{F}\right]_{(-)^{n}}=0, \quad n=2,3,4, \cdots
\end{aligned}
$$

Next we further introduce infinite ghosts and ghost momenta in addition to those of (4.20) in the purely bosonic model:

$$
\begin{aligned}
& \eta_{2 n}^{F}, \quad \eta_{2 n}^{\prime F}, \quad P_{2 n}^{F}, \quad P_{2 n}^{\prime F} \\
& \eta_{2 n-1}^{B}, \quad \eta_{2 n-1}^{B}, \quad P_{2 n-1}^{B}, \quad P_{2 n-1}^{\prime B}, \quad n=1,2,3, \cdots .
\end{aligned}
$$

Since these fields correspond to the fermionic constraints (4.48) and (4.49) the fields with odd (even) ghost numbers are bosonic (fermionic). Thus we now have $\eta_{n}^{B, F}, \eta_{n}^{B, F}$, $P_{n}^{B, F}$ and $P_{n}^{\prime B, F}$ for all ghost numbers. The system we now consider possesses the same algebraic structure as that of the purely bosonic model because in the generalized Chern-Simons theories fermionic and bosonic fields are treated in a unified manner. Therefore what we have to do is just to replace indices of the ghosts and the ghost momenta which were either even or odd integers in the purely bosonic model to all integers. After these modifications we can show that the same BRST transformations and the gauge-fixed action as those in the Lagrangian formulation are obtained in the Hamiltonian formulation.

\section{Perturbative aspects of the model}

\subsection{Partition function}

In this section we present a perturbative analysis of the quantized gauge-fixed action (3.32). First we investigate the partition function of the model. It is expected that the partition function is simply equal to 1 due to the topological nature of the model, which can be also understood in the Hamiltonian formulation where it is shown that there is no local physical dynamical variables. We show in the following how it is realized in a certain regularization scheme. 
It is easy to see that the partition function is one loop exact, which will be proved in the next subsection by treating higher order corrections generally. In this subsection we evaluate the one loop contribution to the partition function. We have only to extract the quadratic terms from the gauge-fixed action and evaluate the determinant factors coming from the Gaussian path-integrations. In the case of the model containing both bosonic and fermionic fields in the starting classical action treated in section 2 and 3, the bosonic and fermionic fields possess the same kinetic terms (3.33) up to the sign factors due to the Grassmannian property of fermionic fields. Therefore the partition function is trivially 1 since the contribution from the bosonic and fermionic fields cancels in each ghost number sector. Although this can be attributed to a (scalar) supersymmetry of the kinetic terms, it is not a symmetry of the full gauge-fixed action. Thus the topological nature behind the triviality of the partition function is hidden by this symmetry of the kinetic term. On the other hand, in the case of the purely bosonic model treated in the previous paper, the triviality follows from the cancellation among infinite ghost number sectors, which we show in the following.

The quadratic terms of the gauge-fixed action of the purely bosonic model are

$$
\begin{aligned}
S_{k i n}=\int d^{2} x \operatorname{Tr}\{ & -\phi \epsilon^{\mu \nu} \partial_{\mu} \omega_{\nu}+\partial^{\mu} \omega_{\mu} b_{0}+\epsilon_{\mu \nu}^{-1} \partial^{\nu} B b_{0}^{\mu}+\partial_{\mu} \eta_{0} b_{0}^{\mu} \\
& +\sum_{n=1}^{\infty}\left(-\partial^{\mu} \bar{C}_{n} \partial_{\mu} C_{n}-\frac{1}{2}\left(\partial^{\mu} \bar{C}_{n}^{\nu}-\partial^{\nu} \bar{C}_{n}^{\mu}\right)\left(\partial_{\mu} C_{n \nu}-\partial_{\nu} C_{n \mu}\right)\right) \\
& \left.+\sum_{n=1}^{\infty}\left(\partial^{\mu} C_{n \mu} b_{n}+\epsilon_{\mu \nu}^{-1} \partial^{\nu} \widetilde{C}_{n} b_{n}^{\mu}+\partial_{\mu} \eta_{n-1} b_{n-1}^{\mu}-\partial_{\mu} \bar{C}_{n}^{\mu} \pi_{n}\right)\right\}
\end{aligned}
$$

where we adopt the notation in the previous paper and the fields of odd (even) ghost number are fermionic (bosonic). It is straightforward to find the contributions to the partition function $Z$ from each ghost number sector. Path-integral over fields of ghost number $\pm n \neq 0$ gives $\left(\operatorname{det} \triangle^{(0)}\right)^{4 \epsilon_{n}}$ where $\epsilon_{n}$ is $+1(-1)$ for odd $n$ (even $n$ ) while fields of ghost number 0 contribute $\left(\operatorname{det} \triangle^{(0)}\right)^{-2}$. Here $\triangle^{(0)}$ is the Laplacian for zero forms and we have used the relation of the Laplacian for one forms $\operatorname{det} \triangle^{(1)}=\left(\operatorname{det} \triangle^{(0)}\right)^{2}$. Thus the log of the partition function becomes

$$
\ln Z=-4 \ln \operatorname{det} \triangle^{(0)} \times\left(\frac{1}{2}+\sum_{n=1}^{\infty}(-)^{n}\right) .
$$

The contribution from the ghost number zero sector is twice overcancelled by that from the ghost number \pm 1 sector and the sum is again overcancelled by that from the ghost number \pm 2 sector, and so on. This reflects the structure of the reducibility of the model 
that the number of gauge and reducibility parameters are twice the real gauge degrees of freedom which can actually gauge away all local dynamical variables. The gaugefixed action is defined as an infinite series which possesses the BRST invariance, which implies that the contribution from the ghost number zero sector should be canceled by the sum of contributions from nonzero ghost number sectors. Therefore we should require that the sum in eq.(5.1) be zero as a regularization for the summation over infinite ghost number sectors. This can be accomplished by the zeta function regularization,

which leads to $\sum_{n=1}^{\infty}(-)^{n}=-\frac{1}{2}$. We adopt the zeta function regularization henceforth as a regularization for the summation of infinite series of ghost contributions.

It should be noted that though we have discussed the triviality of the partition function in the space with flat metric, the same arguments hold in curved spaces except for the contribution from the zero mode due to the global structure of the space.

\section{$5.2 \quad$ Higher order corrections}

In this subsection we investigate loop effects of the gauge-fixed action (3.32). It is convenient to path-integrate out the auxiliary fields $b_{n}, \eta_{n}$ and $\pi_{n}$, which imposes the Landau gauge conditions

$$
\begin{array}{lll}
\partial^{\mu} C_{n \mu}^{F}=0, & \partial_{\mu} b_{n}^{F \mu}=0, & \partial_{\mu} \bar{C}_{n}^{F \mu}=0, \\
\partial^{\mu} C_{n \mu}^{B}=0, & \partial_{\mu} b_{n}^{B \mu}=0, & \partial_{\mu} \bar{C}_{n}^{B \mu}=0 .
\end{array}
$$

We introduce the following compact notation

$$
\begin{aligned}
C^{F} & =\sum_{n=-\infty}^{\infty} C_{n}^{F}, & C^{B} & =\sum_{n=-\infty}^{\infty} C_{n}^{B}, \\
C_{\mu}^{F} & =\sum_{n=-\infty}^{\infty} C_{\mu n}^{F}, & C_{\mu}^{B} & =\sum_{n=-\infty}^{\infty} C_{\mu n}^{B}, \\
\widetilde{C}^{F} & =\sum_{n=-\infty}^{\infty} \widetilde{C}_{n}^{F}, & \widetilde{C}^{B} & =\sum_{n=-\infty}^{\infty} \widetilde{C}_{n}^{B}, \\
b^{F \mu} & =\sum_{n=0}^{\infty} b_{n}^{F \mu}, & b^{B \mu} & =\sum_{n=0}^{\infty} b_{n}^{B \mu} .
\end{aligned}
$$

Here the fields with negative ghost number indices are

$$
\begin{aligned}
& C_{-n}^{F}=\epsilon_{\mu \nu}^{-1} \partial^{\mu} \bar{C}_{n}^{F \nu}, \quad C_{-n}^{B}=-\epsilon_{\mu \nu}^{-1} \partial^{\mu} \bar{C}_{n}^{B \nu}, \\
& C_{\mu-n}^{F}=-\epsilon_{\mu \nu}^{-1} \partial^{\nu} \bar{C}_{n}^{F}, \quad C_{\mu-n}^{B}=-\epsilon_{\mu \nu}^{-1} \partial^{\nu} \bar{C}_{n}^{B}, \\
& \widetilde{C}_{-n}^{F}=0, \quad \widetilde{C}_{-n}^{B}=0, \quad n=1,2,3, \cdots,
\end{aligned}
$$


where the identifications (3.25) and (3.31) are used. Then the gauge-fixed action (3.32) is rewritten in the form

$$
\begin{aligned}
S_{t o t}=- & \int d^{2} x \mathrm{Gh}^{0}\left\{\eta_{a b} \epsilon^{\mu \nu}\left(C_{\mu}^{B a} \partial_{\nu} C^{B b}+C_{\mu}^{F a} \partial_{\nu} C^{F b}\right)+\eta_{a b} \epsilon_{\mu \nu}^{-1}\left(\partial^{\nu} \widetilde{C}^{B a} b^{B b \mu}+\partial^{\nu} \widetilde{C}^{F a} b^{F b \mu}\right)\right. \\
& +F_{a b c}\left(\frac{1}{2} \epsilon^{\mu \nu} C_{\mu}^{B a} C_{\nu}^{B b} C^{B c}+\epsilon^{\mu \nu} C_{\mu}^{F a} C_{\nu}^{B b} C^{F c}+C^{B a} C^{F b} \widetilde{C}^{F c}+\frac{1}{2} C^{F a} C^{F b} \widetilde{C}^{B c}\right) \\
& \left.+D_{a b c}\left(-\frac{1}{2} \epsilon^{\mu \nu} C_{\mu}^{F a} C_{\nu}^{F b} C^{B c}+\frac{1}{2} C^{B a} C^{B b} \widetilde{C}^{B c}\right)\right\}
\end{aligned}
$$

where $F_{a b c}=\operatorname{Tr}\left(T_{a}\left[T_{b}, T_{c}\right]\right)=\eta_{a d} f_{b c}^{d}$ is a totally antisymmetric structure constant while $D_{a b c}=\operatorname{Tr}\left(T_{a}\left\{T_{b}, T_{c}\right\}\right)=\eta_{a d} h_{b c}^{d}$ is a totally symmetric structure constant. $\mathrm{Gh}^{0}$ represents to pick up the zero ghost number terms as in eq.(3.16). Since the field $b^{B \mu}$ and $b^{F \mu}$ do not interact with other fields, the perturbation theory is much simplified.

From this expression and the Landau gauge conditions (5.2), we can obtain propagators

$$
\begin{array}{r}
\left\langle C_{-n}^{G a}(x) C_{m \mu}^{G b}(0)\right\rangle=\int \frac{d^{2} p}{(2 \pi)^{2}} e^{i p x} \frac{-\epsilon_{\mu \nu}^{-1} p^{\nu}}{p^{2}} \delta_{n m}\left(\eta^{-1}\right)^{a b} \equiv D_{\mu}(x) \delta_{n m}\left(\eta^{-1}\right)^{a b}, \\
\left\langle b_{n}^{G a \nu}(x) \widetilde{C}_{m}^{G b}(0)\right\rangle=\int \frac{d^{2} p}{(2 \pi)^{2}} e^{i p x} \frac{-\epsilon^{\mu \nu} p_{\nu}}{p^{2}} \delta_{n m}\left(\eta^{-1}\right)^{a b} \equiv \widetilde{D}^{\mu}(x) \delta_{n m}\left(\eta^{-1}\right)^{a b}, \\
G=B, F,
\end{array}
$$

which imply the propagators for the component fields:

$$
\begin{aligned}
\left\langle\phi^{a}(x) \omega_{\mu}^{b}(0)\right\rangle & =\left\langle\chi^{a}(x) \chi_{\mu}^{b}(0)\right\rangle=D_{\mu}(x)\left(\eta^{-1}\right)^{a b} \\
\left\langle C_{n \mu}^{G a}(x) \bar{C}_{m}^{G b \nu}(0)\right\rangle & =-i \int \frac{d^{2} p}{(2 \pi)^{2}} e^{i p x}\left(\delta_{\mu}^{\nu}-\frac{p_{\mu} p^{\nu}}{p^{2}}\right) \frac{1}{p^{2}} \delta_{n m}\left(\eta^{-1}\right)^{a b} \\
\left\langle C_{n}^{G a}(x) \bar{C}_{m}^{G b}(0)\right\rangle & =-i \int \frac{d^{2} p}{(2 \pi)^{2}} e^{i p x} \frac{1}{p^{2}} \delta_{n m}\left(\eta^{-1}\right)^{a b} \\
\left\langle b_{n}^{G a \mu}(x) \widetilde{C}_{m}^{G b}(0)\right\rangle & =\widetilde{D}^{\mu}(x) \delta_{n m}\left(\eta^{-1}\right)^{a b}
\end{aligned}
$$

We now investigate the effective action obtained as a sum of 1PI diagrams. For a 1PI graph contributing to the effective action we denote the numbers of external legs as $E_{C}, E_{C_{\mu}}$ and $E_{\widetilde{C}}$ for $C^{G}, C_{\mu}^{G}$ and $\widetilde{C}^{G}$, respectively, the number of propagators as $P$ and the number of loops as $L$. As for vertices, we classify them into two categories, $C-C_{\mu}-C_{\nu}$ type and $C-C-\widetilde{C}$ type, and denote the numbers of vertices as $V$ and

${ }^{*}$ We take the flat Minkowski metric $g_{\mu \nu}=\operatorname{diag}(-1,+1)$. The propagators are thus obtained by the Minkowskian path-integral.

${ }^{\dagger}$ We do not discriminate fermionic and bosonic fields here. Thus $E_{C}$ is the sum of the number of external legs for $C^{F}$ and $C^{B}$, for example. 
$\widetilde{V}$, respectively. Then the following relations hold:

$$
\begin{aligned}
E_{\widetilde{C}} & =\widetilde{V}, \\
V+2 \widetilde{V} & =P+E_{C}, \\
2 V & =P+E_{C_{\mu}}, \\
L & =P-(V+\widetilde{V})+1 .
\end{aligned}
$$

From these relations we obtain

$$
L=E_{\widetilde{C}}-E_{C}+1
$$

which shows that multiloop graphs must be accompanied by external legs of $\widetilde{C}$. In particular the partition function is one loop exact as discussed in the previous subsection. We further obtain the superficial degree of divergence

$$
D=2 L-P=2-2 E_{\widetilde{C}}-E_{C_{\mu}}
$$

This implies that the possible ultraviolet divergences exist only for $L=1, E_{C_{\mu}}=1,2$, $E_{C}=E_{\widetilde{C}}=0 ; L=1, E_{\widetilde{C}}=E_{C}=1, E_{C_{\mu}}=0$ and $L=2, E_{\widetilde{C}}=1, E_{C}=E_{C_{\mu}}=0$ besides the partition function, which we have shown to be one. In the following we will see that all these contributions actually vanish in the regularization scheme used for the partition function and thus the theory is free from the ultraviolet divergence.

First we examine one loop diagrams with two external legs. For diagrams with two bosonic external fields, $C_{m \mu}^{B a}\left(x_{1}\right)$ and $C_{-m \nu}^{B a^{\prime}}\left(x_{2}\right)$ or $C_{m}^{B a}\left(x_{1}\right)$ and $\widetilde{C}_{-m}^{B a^{\prime}}\left(x_{2}\right)$, there are two types of graphs: those with a loop of bosonic fields and of fermionic fields. Each graph gives the same contribution except for the sign, i.e., a fermion loop gives an extra sign factor. Thus bosonic loops and fermionic loops cancel with each other. We have two comments in order. First each contribution itself is divergent. For example a bosonic loop with the first type of external legs yields

$$
\begin{gathered}
\int d^{2} x_{1} d^{2} x_{2} C_{m \mu}^{B a}\left(x_{1}\right) C_{-m \nu}^{B a^{\prime}}\left(x_{2}\right) F_{a b c} F_{a^{\prime} b^{\prime} c^{\prime}}\left(\eta^{-1}\right)^{b b^{\prime}}\left(\eta^{-1}\right)^{c c^{\prime}} \\
(-i)^{2} \epsilon^{\mu \mu^{\prime}} \epsilon^{\nu \nu^{\prime}} D_{\mu^{\prime}}\left(x_{1}-x_{2}\right) D_{\nu^{\prime}}\left(x_{2}-x_{1}\right)
\end{gathered}
$$

which is logarithmically divergent to be consistent with the superficial degree of divergence. The same regularization should be applied both for bosonic and fermionic loops, which makes the whole contribution vanish. The second comment is for the case of purely bosonic models. In this case bosonic loops and fermionic loops appear 
alternatively according to the ghost number of the fields of the loop. Thus the cancellation does not work at each fixed ghost number sector and the whole contribution is $\sum_{n=-\infty}^{\infty}(-)^{n}$ multiplied by a bosonic loop contribution of the form (5.10). This is reminiscent of the case for the partition function. We should adopt the zeta function regularization for the summation to have $\sum_{n=-\infty}^{\infty}(-)^{n}=0$. This is closely related with the BRST invariance of the theory. Indeed if it were not set to zero, the BRST Ward-Takahashi identity $\left\langle\delta_{B} C_{-1}\right\rangle=0$ would be violated.

For diagrams with two fermionic external fields, $C_{m \mu}^{F a}\left(x_{1}\right)$ and $C_{-m \nu}^{F a^{\prime}}\left(x_{2}\right)$ or $C_{m}^{F a}\left(x_{1}\right)$ and $\widetilde{C}_{-m}^{F a^{\prime}}\left(x_{2}\right)$, there are two graphs for each ghost number of the loop fields: a field of ghost number $n+m$ can be bosonic or fermionic. These contribution, however, does not cancel with each other. For example a diagram with the first type of external fields gives the contribution

$$
\begin{gathered}
\int d^{2} x_{1} d^{2} x_{2} C_{m \mu}^{F a}\left(x_{1}\right) C_{-m \nu}^{F a^{\prime}}\left(x_{2}\right) F_{a b c} D_{a^{\prime} b^{\prime} c^{\prime}}\left(\eta^{-1}\right)^{b b^{\prime}}\left(\eta^{-1}\right)^{c c^{\prime}} \\
(-i)^{2} \epsilon^{\mu \mu^{\prime}} \epsilon^{\nu \nu^{\prime}} D_{\mu^{\prime}}\left(x_{1}-x_{2}\right) D_{\nu^{\prime}}\left(x_{2}-x_{1}\right) .
\end{gathered}
$$

It vanishes, however, by itself due to the totally symmetric and antisymmetric property of $D_{a b c}$ and $F_{a b c}$. Thus we have shown one loop diagrams with two external legs vanish completely.

Next we investigate two loop tadpole diagrams with an external $\widetilde{C}^{G}$. Due to the ghost number conservation and Grassmannian property, the only possible contribution appears for $\widetilde{C}_{0}^{B}=B$. It vanishes, however, since the two loop tadpole diagrams contain one loop subdiagrams with two external legs which vanish as we have shown above. Finally the one loop tadpole with an external $C_{\mu}^{G}$ vanishes due to the Lorentz invariance of the loop integration. Thus we have shown that all possibly divergent diagrams vanish.

Although the generalized Chern-Simons theory is free from the ultraviolet divergence, there appear infinite quantities to be taken care of due to the existence of the infinite fields. As an illuminating example we consider a one loop diagram with three external legs. For diagrams with external legs $C_{\mu}^{B} C_{\nu}^{B} C_{\lambda}^{B}$ or $C_{\mu}^{B} C^{B} \widetilde{C}^{B}$, contributions from bosonic loops and fermionic loops cancel with each other as in the previous case. For diagrams with external $\operatorname{legs} C_{\mu}^{B} C_{\nu}^{F} C_{\lambda}^{F}$ or $C_{\mu}^{B} C^{F} \widetilde{C}^{F}$, however, cancellations among diagrams or vanishing due to the group theoretic property do not occur. For example 
we have

$$
\begin{aligned}
& \int d^{2} x_{1} d^{2} x_{2} d^{2} x_{3} C_{m+n \mu}^{B a_{1}}\left(x_{1}\right) C_{-m \nu}^{F a_{2}}\left(x_{2}\right) C_{-n \lambda}^{F a_{3}}\left(x_{3}\right) \\
&\left(\eta^{-1}\right)^{b b^{\prime}}\left(\eta^{-1}\right)^{c c^{\prime}}\left(\eta^{-1}\right)^{d d^{\prime}}\left(F_{b^{\prime} a_{1} c} F_{c^{\prime} a_{2} d} D_{d^{\prime} a_{3} b}-F_{b^{\prime} a_{1} c} D_{c^{\prime} a_{2} d} F_{d^{\prime} a_{3} b}\right) \\
& \epsilon^{\mu \mu^{\prime}} \epsilon^{\nu \nu^{\prime}} \epsilon^{\lambda \lambda^{\prime}} D_{\mu^{\prime}}\left(x_{1}-x_{2}\right) D_{\nu^{\prime}}\left(x_{2}-x_{3}\right) D_{\lambda^{\prime}}\left(x_{3}-x_{1}\right) \sum_{k=-\infty}^{\infty} 1,
\end{aligned}
$$

as the first type of contribution. The summation $\sum_{k=-\infty}^{\infty} 1$ is ambiguous even using the zeta function regularization since the summation extends both plus and minus infinity. This appears to imply that the gauge-fixed action (3.32) is not fully consistent at the quantum level since we do not have counter terms to control those divergences. However we may be able to regard them as gauge-fixing artifacts. The point is that the divergent 1PI diagrams contribute only to unphysical correlation functions. In the present analysis with the flat metric, the only physical variable is the zero mode of $\phi$ field and other BRST singlet operators are trivial ones. Thus physical correlation functions which are independent of gauge-fixing conditions are zero and thus free from divergence. Therefore there may exist better gauge-fixing conditions which yield finite correlation functions also for the unphysical sector. Keeping this in mind, we can

take $\sum_{k=-\infty}^{\infty} 1$ as an integer value $N$ by the zeta function regularization, which leaves the value of $N$ arbitrary. Then physical correlation functions are independent of $N$ while unphysical ones become finite. It should be noted that BRST Ward-Takahashi identities are satisfied after this regularization. These are the perturbative aspects of the gauge-fixed action (3.32). It is an open question whether it gives interesting information even with this sick property in the unphysical sector.

\section{Quantization of even-dimensional models}

\subsection{Four-dimensional model}

The arguments of infinite reducibility given in subsection 3.1 for the two-dimensional model are applicable to any even-dimensional models of generalized Chern-Simons actions with the minor change of introducing higher form gauge parameters. In other words the infinite reducibility is a common feature of the generalized Chern-Simons actions in any dimensions even including odd dimensions. Furthermore the procedure of constructing the BRST transformation and gauge-fixed action is dimension independent and thus applicable to any even-dimensional models. In this subsection we present 
the quantization of the purely bosonic four-dimensional model by the Lagrangian formulation, explicitly, and show how the quantization of the four-dimensional model goes parallel to the case of the two-dimensional model.

The four-dimensional action without fermionic gauge fields is given in eq.(2.16). The corresponding gauge transformations without fermionic gauge parameters $(\alpha=\hat{\alpha}=0)$ are given by

$$
\begin{aligned}
\delta \phi & =[\phi, v], \\
\delta \omega & =d v+[\omega, v]-\{\phi, u\}, \\
\delta B & =d u+\{\omega, u\}+[B, v]+[\phi, b], \\
\delta \Omega & =d b+[\omega, b]+[\Omega, v]-\{B, u\}+[\phi, b], \\
\delta H & =d U+\{\omega, U\}+\{\Omega, u\}+[H, v]+[B, b]+[\phi, V],
\end{aligned}
$$

where we have introduced the following notation:

$$
\begin{aligned}
\mathcal{V} & =\mathbf{1} \hat{a}+\mathbf{i} a \\
& \equiv \mathbf{1}(v+b+V)+\mathbf{i}(u+U)
\end{aligned}
$$

with $v, u, b, U, V$ being 0-, 1-, 2-, 3-, 4-form bosonic gauge parameters, respectively.

We now introduce generalized variables completely parallel to the two-dimensional eqs.(3.4) and (3.5):

$$
\begin{aligned}
\mathcal{V}_{2 n}=\mathbf{j}\left(u_{2 n}+U_{2 n}\right)+\mathbf{k}\left(v_{2 n}+b_{2 n}+V_{2 n}\right) \in \Lambda_{-}, & \\
\mathcal{V}_{2 n+1}=\mathbf{1}\left(v_{2 n+1}+b_{2 n+1}+V_{2 n+1}\right)-\mathbf{i}\left(u_{2 n+1}+U_{2 n+1}\right) \in \Lambda_{+}, & \\
& n=0,1,2, \cdots .
\end{aligned}
$$

By applying the above definitions of generalized variables $\mathcal{V}_{2 n}$ and $\mathcal{V}_{2 n+1}$ to eqs.(3.6) and (3.7), we can show the infinite reducibility of the four-dimensional model based on the same arguments of two-dimensional case.

Next we need to introduce infinite series of generalized fields corresponding to the generalized variables:

$$
C_{n}, \quad C_{n \mu}, \quad \frac{1}{2 !} C_{n \mu \nu}, \quad \frac{1}{3 !} C_{n \mu \nu \rho}, \quad \frac{1}{4 !} C_{n \mu \nu \rho \sigma}
$$

where the index $n$ indicates the ghost number of the fields and the fields with even (odd) ghost number are bosonic (fermionic). The fields with ghost number 0 are the 
classical fields

$$
C_{0}=\phi, \quad C_{0 \mu}=\omega_{\mu}, \quad C_{0 \mu \nu}=B_{\mu \nu}, \quad C_{0 \mu \nu \rho}=\Omega_{\mu \nu \rho}, \quad C_{0 \mu \nu \rho \sigma}=H_{\mu \nu \rho \sigma} .
$$

Then we redefine a generalized gauge field

$$
\begin{aligned}
\widetilde{\mathcal{A}}=\mathbf{1} \psi & +\mathbf{i} \hat{\psi}+\mathbf{j} A+\mathbf{k} \hat{A} \in \Lambda_{-} \\
\psi & =\sum_{n=-\infty}^{\infty}\left(C_{2 n+1 \mu} d x^{\mu}+\frac{1}{3 !} C_{2 n+1 \mu \nu \rho} d x^{\mu} \wedge d x^{\nu} \wedge d x^{\rho}\right) \\
\hat{\psi} & =\sum_{n=-\infty}^{\infty}\left(C_{2 n+1}+\frac{1}{2 !} C_{2 n+1 \mu \nu} d x^{\mu} \wedge d x^{\nu}+\frac{1}{4 !} C_{2 n+1 \mu \nu \rho \sigma} d x^{\mu} \wedge d x^{\nu} \wedge d x^{\rho} \wedge d x^{\sigma}\right), \\
A & =\sum_{n=-\infty}^{\infty}\left(C_{2 n \mu} d x^{\mu}+\frac{1}{3 !} C_{2 n \mu \nu \rho} d x^{\mu} \wedge d x^{\nu} \wedge d x^{\rho}\right), \\
\hat{A} & =\sum_{n=-\infty}^{\infty}\left(C_{2 n}+\frac{1}{2 !} C_{2 n \mu \nu} d x^{\mu} \wedge d x^{\nu}+\frac{1}{4 !} C_{2 n \mu \nu \rho \sigma} d x^{\mu} \wedge d x^{\nu} \wedge d x^{\rho} \wedge d x^{\sigma}\right),
\end{aligned}
$$

where we have explicitly shown the differential form dependence.

The definition of the generalized antibracket defined in subsection 3.2 is universal in any even dimensions as far as the generalized gauge field is properly defined as an element of $\Lambda_{-}$class. Using the above definitions of generalized gauge fields, we claim that the same form of action as in two dimensions

$$
S_{\text {min }}=\int \operatorname{Tr}_{\mathbf{k}}^{0}\left(\frac{1}{2} \widetilde{\mathcal{A}} Q \widetilde{\mathcal{A}}+\frac{1}{3} \widetilde{\mathcal{A}}^{3}\right)
$$

is the minimal part of quantized action in four dimensions. There is a natural procedure to derive BRST transformation, to prove nilpotency of BRST transformation and to show that $S_{\text {min }}$ satisfies master equation, by using the generalized antibracket arguments of subsection 3.2. The BRST transformations and the nilpotency have the same form as (3.23) and (3.24)

$$
\begin{aligned}
\delta_{\lambda} \widetilde{\mathcal{A}} & \equiv(\widetilde{\mathcal{A}}, \widetilde{S})_{\lambda, \mathbf{k}}=-\widetilde{\mathcal{F}} \mathbf{i} \lambda, \\
s^{2} \widetilde{\mathcal{A}} \lambda_{2} \lambda_{1} & \equiv \delta_{\lambda_{2}} \delta_{\lambda_{1}} \widetilde{\mathcal{A}}=0 .
\end{aligned}
$$

We can then show that $S_{\text {min }}$ satisfies the master equation

$$
\delta_{\lambda} S_{\text {min }}=\left(S_{\text {min }}, S_{\text {min }}\right)_{\lambda, \mathbf{k}}=\left(S_{\text {min }}, S_{\text {min }}\right) \cdot \lambda=0
$$

where $($,$) is the original antibracket defined by (3.9) with the following identifications$ of antifields:

$$
\frac{1}{4 !} \epsilon^{\mu \nu \rho \sigma} C_{-2 n+1 \mu \nu \rho \sigma}=C_{2(n-1)}^{*}, \quad \frac{1}{4 !} \epsilon^{\mu \nu \rho \sigma} C_{-2 n \mu \nu \rho \sigma}=-C_{2 n-1}^{*},
$$




$$
\begin{array}{rlrl}
\frac{1}{3 !} \epsilon^{\mu \nu \rho \sigma} C_{-2 n+1 \nu \rho \sigma} & =C_{2(n-1)}^{\mu *}, & & \frac{1}{3 !} \epsilon^{\mu \nu \rho \sigma} C_{-2 n \nu \rho \sigma}=C_{2 n-1}^{\mu *}, \\
\frac{1}{2 !} \epsilon^{\mu \nu \rho \sigma} C_{-2 n+1 \rho \sigma} & =-C_{2(n-1)}^{\mu \nu *}, & \frac{1}{2 !} \epsilon^{\mu \nu \rho \sigma} C_{-2 n \rho \sigma}=-C_{2 n-1}^{\mu \nu *}, \\
\epsilon^{\mu \nu \rho \sigma} C_{-2 n+1 \sigma} & =C_{2(n-1)}^{\mu \nu \rho *}, & \epsilon^{\mu \nu \rho \sigma} C_{-2 n \sigma}=C_{2 n-1}^{\mu \nu \rho *}, \\
\epsilon^{\mu \nu \rho \sigma} C_{-2 n+1} & =C_{2(n-1)}^{\mu \nu \rho \sigma *}, & & \epsilon^{\mu \nu \rho \sigma} C_{-2 n}=-C_{2 n-1}^{\mu \nu \rho \sigma *} .
\end{array}
$$

For completeness we give the explicit forms of the BRST transformations for the component fields in the appendix.

In order to fix the gauge we introduce the nonminimal action

$$
\begin{aligned}
S_{n o n m i n}=\int d^{4} x \sum_{n=1}^{\infty} \operatorname{Tr}( & \bar{C}_{n \mu \nu \rho}^{*} b_{n-1}^{\mu \nu \rho}+\bar{C}_{n \mu \nu}^{*} b_{n-1}^{\mu \nu}+\bar{C}_{n \mu}^{*} b_{n-1}^{\mu}+\bar{C}_{n}^{*} b_{n-1} \\
& +\eta_{n-1}^{\mu \nu *} \pi_{n \mu \nu}+\eta_{n-1}^{\mu *} \pi_{n \mu}+\eta_{n-1}^{*} \pi_{n} \\
& \left.+C_{n}^{* \mu} \rho_{n-1 \mu}+\xi_{n}^{*} \rho_{n-1}+\zeta_{n-1}^{*} \sigma_{n}\right)
\end{aligned}
$$

where the ghost number of nonminimal fields is $n$ for $\eta_{n}, \eta_{n}^{\mu}, \eta_{n}^{\mu \nu}, \pi_{n}, \pi_{n \mu}$ and $\zeta_{n}$, and $-n$ for $\bar{C}_{n}, \bar{C}_{n}^{\mu}, \bar{C}_{n}^{\mu \nu}, \bar{C}_{n}^{\mu \nu \rho}, b_{n}, b_{n}^{\mu}, b_{n}^{\mu \nu}, b_{n}^{\mu \nu \rho}, \xi_{n}$ and $\xi_{n}^{\mu}$, respectively. The BRST transformations of these fields are defined by the nonminimal action, as usual. In order to impose the Landau type gauge-fixing condition for the antisymmetric tensor fields in each sector of the ghost number, we adopt the gauge fermion

$$
\begin{aligned}
\Psi=\int d^{4} x \sum_{n=1}^{\infty} \operatorname{Tr}( & \bar{C}_{n}^{\mu \nu \rho} \partial^{\sigma} C_{n-1 \mu \nu \rho \sigma}+\bar{C}_{n}^{\mu \nu} \partial^{\rho} C_{n-1 \mu \nu \rho}+\bar{C}_{n}^{\mu} \partial^{\nu} C_{n-1 \mu \nu}+\bar{C}_{n} \partial^{\mu} C_{n-1 \mu} \\
& +\bar{C}_{n}^{\mu \nu \rho} \partial_{\mu} \eta_{n-1 \nu \rho}+\bar{C}_{n}^{\mu \nu} \partial_{\mu} \eta_{n-1 \nu}+\bar{C}_{n}^{\mu} \partial_{\mu} \eta_{n-1} \\
& \left.+\xi_{n}^{\mu} \partial^{\nu} \eta_{n-1 \mu \nu}+\xi_{n} \partial^{\mu} \eta_{n-1 \mu}+\zeta_{n-1} \partial_{\mu} \xi_{n}^{\mu}\right) .
\end{aligned}
$$

Eliminating the antifields by $\Phi_{A}^{*}=\frac{\partial \Psi}{\partial \Phi^{A}}$, we obtain the complete form of the fourdimensional quantized gauge-fixed action.

\subsection{General features in the quantization of arbitrary even- dimensional models}

As we have shown in the previous subsection, the quantization procedure in two dimensions and four dimensions goes exactly parallel with minor modifications of introducing new fields in higher dimensions. In other words if we try to formulate the quantization procedure in terms of the generalized gauge fields and parameters, it is dimension independent. In order to stress this point we list the general procedure of quantizing the even-dimensional generalized Chern-Simons actions by Lagrangian formulation. 
1. We first define generalized gauge field $\mathcal{A}$ and parameter $\mathcal{V}$ of the form of (2.1) and (2.2) in terms of component fields explicitly as in (2.14) and (6.2).

2. We then obtain concrete forms of, even-dimensional bosonic action $S_{e}^{b}$ of (2.10), gauge transformations (2.11) and equations of motion (2.13), in terms of component fields.

3. We introduce generalized variables $\mathcal{V}_{2 n}$ and $\mathcal{V}_{2 n+1}$ as in (3.4), (3.5) and (6.3), (6.4). Then infinite reducibility is a natural consequence of the relations (3.6) and (3.7).

4. Corresponding to the infinite reducibility, we introduce infinite series of generalized fields as in (3.14) and (6.5) and redefine the generalized gauge field as in (3.15) and (6.6).

5. The minimal action $S_{\text {min }}$ can be defined in terms of the generalized gauge fields as in (3.16) and (6.7).

6. The BRST transformation is given by $s \widetilde{\mathcal{A}}=-\widetilde{\mathcal{F}} \mathbf{i}$ and its nilpotency can be shown by (3.24).

7. $S_{\min }$ satisfies master equation as in (3.26) under the identification that the negative ghost number fields are identified with antifields in a proper way as in (3.25) and $(6.8)$.

8. The nonminimal action $S_{\text {nonmin }}$ and the gauge fermion $\Psi$ for Landau type gaugefixing can be obtained as a natural extension of two- and four- dimensional expressions (3.28), (3.30) and (6.9), (6.10).

9. Substituting the antifields by $\Phi_{A}^{*}=\frac{\partial \Psi}{\partial \Phi^{A}}$ into $S_{\text {min }}+S_{\text {nonmin }}$, we obtain the complete form of the quantized gauge-fixed action.

The quantization of Hamiltonian formulation in arbitrary even dimensions will be carried out in the similar way as in the two-dimensional case. In general the quantizations by the Hamiltonian formulation and the Lagrangian formulation should give the same result. We have explicitly shown the equivalence in two dimensions. For the generalized Chern-Simons actions this point was a priori not clear because of the regurality violation. It was, however, explicitly shown that the regularity violating 
constraints can be used as first class constraints in the Hamiltonian formulation and the result coincides with that of Lagrangian formulation.

In the process of the Hamiltonian quantization we have found several interesting facts. The first class constraints of Hamiltonian formulation are infinitely reducible and thus we need to introduce infinite series of ghosts and ghost momenta to quantize the system. If we properly define a Hamiltonian version of generalized gauge field $\widetilde{\mathcal{A}}$ as in the two-dimensional case (4.21), we can define the same form of BRST differential (4.22) as that of Lagrangian formulation. What is further surprising is that the BRST charge has again the Chern-Simons form of the fermionic sector as can be seen in (4.30).

We can generalize this result in the following: We take an even-dimensional bosonic action as classical action

$$
S_{e}^{b}=\int_{M_{d}} \operatorname{Tr}_{\mathbf{k}}\left(\frac{1}{2} \mathcal{A} Q \mathcal{A}+\frac{1}{3} \mathcal{A}^{3}\right)
$$

we then obtain the quantized minimal action of Lagrangian formulation

$$
S_{\text {min }}=\int_{M_{d}} \operatorname{Tr}_{\mathbf{k}}^{0}\left(\frac{1}{2} \widetilde{\mathcal{A}} Q \widetilde{\mathcal{A}}+\frac{1}{3} \widetilde{\mathcal{A}}^{3}\right)
$$

where we should take ghost number zero sector. The minimal version of BRST charge in the Hamiltonian formulation for the even-dimensional generalized Chern-Simons action is again an generalized Chern-Simons action which is an odd-dimensional fermionic action and should be one dimension lower than the minimal action $S_{\min }$

$$
\Omega_{\text {min }}=\int_{M_{d-1}} \operatorname{Tr}_{\mathbf{1}}^{1}\left(\frac{1}{2} \widetilde{\mathcal{A}} Q^{\prime} \widetilde{\mathcal{A}}+\frac{1}{3} \widetilde{\mathcal{A}}^{3}\right)
$$

where we should take the ghost number one sector. In more general cases for a graded Lie algebra, we must take Htr instead of the simple trace Tr in the above.

Although it is outside of the scope of this paper, we claim that the odd-dimensional case goes completely parallel to the above even-dimensional case with the replacements of the above expressions by $\mathbf{k} \rightarrow \mathbf{j}, \mathbf{1} \rightarrow \mathbf{i}$ and $\operatorname{Tr} \rightarrow$ Str, respectively.

\section{Conclusions and discussions}

We have investigated the quantization of the even-dimensional version of the generalized Chern-Simons actions by the Lagrangian and Hamiltonian formulations. We have found that the models formulated by the generalized Chern-Simons actions are in general infinitely reducible and thus we need to introduce the infinite series of ghost fields 
and the quantizations turn out to be highly nontrivial. It is, however, an important characteristic of the generalized Chern-Simons formulation that the generalized gauge field can accommodate the infinite number of fields in a compact form and treat them in a unified way. We have then found the solution of the master equation in the Lagrangian formulation, the BRST invariant minimal action, which has again the same Chern-Simons form as the starting classical action. With careful considerations of degrees of freedom, we have obtained the gauge-fixed actions by defining gauge fermions with Landau type gauge-fixings. In the Hamiltonian formulation we have found two first class constraints which break Dirac's regularity condition but lead to the same action as that of Lagrangian formulation under a proper choice of the gauge fermion and identifications of the fields.

The quantizations of the models have been successfully carried out while there remains another question if the introduction of infinite series of the ghost fields does not cause any problems at the quantum level. The generalized Chern-Simons actions are in general expected to be topological since the classical actions have the same number of gauge fields and parameters and thus all the gauge fields could be gauged away. The story is, however, not so simple because of the infinite reducibility. As we have shown in section 5 , the infinite series of ghost fields cancel out the quantum effects and then the partition function becomes numerically one and thus the topological nature is kept even at the quantum level. In formulating these contributions from an infinite number of ghosts, we have used zeta function regularization. The correlation functions have the similar nature as the partition function but have some possible problem in the unphysical sector of loop contributions because of the infinite number of the ghost fields. Since the problem appears only in the unphysical sector the models are still expected to be consistent at the quantum level. There remain, however, some open problems in the unphysical sector, which might be related with the regularization problem.

As we have shown in section 6 the quantization procedure of the generalized ChernSimons actions is dimension independent with minor modifications of introducing new higher form fields in higher dimensions. In other words the minimal part of the quantized action have the same Chern-Simons form and the gauge fermion can be introduced with the similar forms for any even dimensions. We have already suggested that the quantization procedure in odd dimensions will be carried out in a parallel way except that we need to care about the graded Lie algebra with the supertrace Str. The quan- 
tizations of the odd-dimensional generalized Chern-Simons actions, which includes the standard three dimensional Chern-Simons action as a special case, will be given elsewhere [16]. In finding dimension independent formulations the proposed generalized antibracket formulation was helpful to find the BRST transformation, its nilpotency and the solution of the master equation. This formulation is, however, proposed in heuristic bases and thus needs sound mathematical backgrounds which might propose a new aspect of the quantization procedure of the generalized Chern-Simons theory.

It is interesting to consider possibly physical aspects of the introduction of an infinite number of the ghost fields. An immediate consequence is a democracy of ghosts and classical fields, i.e., the classical fields are simply the zero ghost number sector among infinitely many ghost fields and thus the classical gauge fields and ghost fields have no essential difference in the minimal action. Furthermore fermionic and bosonic gauge fields are treated in an equal base and the series of infinite ghosts originated from the classical fermionic and bosonic fields are complimentary. In other words if we only introduce bosonic classical fields in the starting action we need to introduce fermionic fields with odd integer ghost number and bosonic fields with even integer ghost number as in the previous paper [12]. If we introduce the classical fermionic gauge fields as in section 2, the odd and even nature should be reversed for the ghost numbers when introducing the corresponding ghost fields to the fermionic gauge fields. It seems to mean that even the fermionic and bosonic fields have no essential difference in the generalized Chern-Simons theory. In other words fermionic fields, bosonic fields, classical fields and ghost fields are mutually inter-related via the quantization procedure.

Another surprising result which became clear after the quantization of Hamiltonian formulation is that the minimal part of the BRST charge of Hamiltonian quantization is the odd-dimensional fermionic counterpart of the generalized Chern-Simons action. This is again suggesting that the BRST charge of Hamiltonian formulation and the minimal action of Lagraingian formulation are inter-related via the quantization procedure. In these inter-related correspondences the quaternions are again playing the fundamental role to relate fermions, bosons, even dimensions and odd dimensions.

It may be important to note at this stage that the generalized form of the BRST transformation suggests a new type of differential. The BRST transformation $s \widetilde{\mathcal{A}}=$ $-\widetilde{\mathcal{F}} \mathbf{i}$, becomes $s_{l} \widetilde{\mathcal{A}}=-\mathbf{i} \widetilde{\mathcal{F}}$, when it is defined as a left variation in accordance with the exterior derivative. Then this can be written as $\left(-\mathbf{i} s_{l}+\mathbf{j} d\right) \widetilde{\mathcal{A}}+\widetilde{\mathcal{A}}^{2}=0$. This is 
suggesting an existence of a differential into ghost direction. The combined differential of the new differential and the old exterior derivative, $\tilde{Q}=-\mathbf{i} s_{l}+\mathbf{j} d$, provides a flat connection condition which could be the equation of motion of a newly defined "ChernSimons action". The definition of the new differential suggests that ghost is equivalent to a product of the differential form and the quaternion $\mathbf{k}$, which is exactly the result of the previous treatment where the equivalence between the generalized Chern-Simons actions and topological particle field theory actions was shown [17].

In the analyses of the quantization of the generalized Chern-Simons theory with abelian $g l(1, \mathbf{R})$ algebra, it was pointed out that a physical degree of freedom which did not exist at the classical level appeared in the constant part of the zero form field at the quantum level due to the violation of the regularity [6]. This situation is unchanged even in the nonabelian cases. We know that the zero form field plays an important role in the generalized Chern-Simons theories as emphasized in the classical discussions [3, 4]. In particular a constant component of the zero form field played a role

of physical order parameter between the gravity and nongravity phases for particular choices of nonabelian gauge algebra. By the analyses of the quantization of Hamiltonian formulation it became clear that the constant mode of the zero form still remains as a physical mode in the quantum level.

As we have stressed in the introduction the success of the quantization of the generalized Chern-Simons actions in even dimensions leads naturally to the quantization of topological gravities in two and four dimensions which were classically well defined [3, 4].

\section{Acknowledgments}

This work is supported in part by Japan Society for the Promotion of Science under the grant number 09640330. 


\section{Appendix A.}

Actions of $D$ and $\stackrel{(k)}{s}$ for all fields

$$
\begin{aligned}
& D P_{2 n}^{B}=\left[P_{2 n}^{B}, \eta_{1}^{F}\right]+\left\{P_{2 n}^{\prime B}, \eta_{1}^{\prime F}\right\}, \\
& D P_{2 n-1}^{F}=\left\{P_{2 n-1}^{F}, \eta_{1}^{F}\right\}+\left\{P_{2 n-1}^{\prime F}, \eta_{1}^{\prime F}\right\}, \\
& D P_{2 n}^{\prime B}=\left[P_{2 n}^{\prime B}, \eta_{1}^{F}\right] \text {, } \\
& D P_{2 n-1}^{\prime F}=\left\{P_{2 n-1}^{\prime F}, \eta_{1}^{F}\right\} \text {, } \\
& \stackrel{(2 k)}{s} \omega_{1}=-\left[P_{2 k}^{B}, \eta_{2 k+1}^{F}\right]-\left\{P_{2 k}^{\prime B}, \eta_{2 k+1}^{\prime F}\right\}, \\
& \stackrel{(2 k-1)}{s} \omega_{1}=-\left\{P_{2 k-1}^{F}, \eta_{2 k}^{B}\right\}-\left[P_{2 k-1}^{\prime F}, \eta_{2 k}^{\prime B}\right] \text {, } \\
& \stackrel{(2 k)}{s} \pi_{\omega_{1}}=-\left[\eta_{2 k+1}^{F}, P_{2 k}^{\prime B}\right] \text {, } \\
& \stackrel{(2 k-1)}{s} \pi_{\omega_{1}}=\left[\eta_{2 k}^{B}, P_{2 k-1}^{\prime F}\right] \text {, } \\
& \stackrel{(2 k)}{s} \eta_{2 n}^{B}=-\left[\eta_{2(n+k)+1}^{F}, P_{2 k}^{\prime B}\right], \\
& \stackrel{(2 k-1)}{s} \eta_{2 n}^{B}=\left[\eta_{2(n+k)}^{B}, P_{2 k-1}^{\prime F}\right] \text {, } \\
& \stackrel{(2 k)}{s} \eta_{2 n-1}^{F}=\left\{\eta_{2(n+k)}^{B}, P_{2 k}^{\prime B}\right\}, \\
& \stackrel{(2 k-1)}{s} \eta_{2 n-1}^{F}=\left\{\eta_{2(n+k)-1}^{F}, P_{2 k-1}^{\prime F}\right\}, \\
& \stackrel{(2 k)}{s} \eta_{2 n}^{\prime B}=-\left[P_{2 k}^{B}, \eta_{2(n+k)+1}^{F}\right]-\left\{P_{2 k}^{\prime B}, \eta_{2(n+k)+1}^{\prime F}\right\}, \\
& \stackrel{(2 k-1)}{s} \eta_{2 n}^{\prime B}=-\left\{P_{2 k-1}^{F}, \eta_{2(n+k)}^{B}\right\}-\left[P_{2 k-1}^{\prime F}, \eta_{2(n+k)}^{\prime B}\right], \\
& \stackrel{(2 k)}{S} \eta_{2 n-1}^{\prime F}=\left[\eta_{2(n+k)}^{B}, P_{2 k}^{B}\right]+\left[\eta_{2(n+k)}^{\prime B}, P_{2 k}^{\prime B}\right] \text {, } \\
& \stackrel{(2 k-1)}{s} \eta_{2 n-1}^{\prime F}=\left\{\eta_{2(n+k)-1}^{F}, P_{2 k-1}^{F}\right\}+\left\{\eta_{2(n+k)-1}^{\prime F}, P_{2 k-1}^{\prime F}\right\}, \\
& \stackrel{(2 k)}{s} P_{2 n}^{B}=\left[P_{2(n+k)}^{B}, \eta_{2 k+1}^{F}\right]+\left\{P_{2(n+k)}^{\prime B}, \eta_{2 k+1}^{\prime F}\right\}, \\
& \stackrel{(2 k-1)}{s} P_{2 n}^{B}=\left\{P_{2(n+k)-1}^{F}, \eta_{2 k}^{B}\right\}+\left[P_{2(n+k)-1}^{\prime F}, \eta_{2 k}^{\prime B}\right] \text {, } \\
& \stackrel{(2 k)}{s} P_{2 n+1}^{F}=\left\{\eta_{2 k+1}^{F}, P_{2(n+k)+1}^{F}\right\}+\left\{\eta_{2 k+1}^{\prime F}, P_{2(n+k)+1}^{\prime F}\right\}, \\
& \stackrel{(2 k-1)}{s} P_{2 n+1}^{F}=\left[\eta_{2 k}^{B}, P_{2(n+k)}^{B}\right]+\left[\eta_{2 k}^{\prime B}, P_{2(n+k)}^{\prime B}\right], \\
& \stackrel{(2 k)}{s} P_{2 n}^{\prime B}=-\left[\eta_{2 k+1}^{F}, P_{2(n+k)}^{\prime B}\right], \\
& \stackrel{(2 k-1)}{s} P_{2 n}^{\prime B}=\left[\eta_{2 k}^{B}, P_{2(n+k)-1}^{\prime F}\right], \\
& \stackrel{(2 k)}{s} P_{2 n+1}^{\prime F}=\left\{\eta_{2 k+1}^{F}, P_{2(n+k)+1}^{\prime F}\right\} \text {, } \\
& \stackrel{(2 k-1)}{s} P_{2 n+1}^{\prime F}=\left\{\eta_{2 k}^{B}, P_{2(n+k)}^{\prime B}\right\} .
\end{aligned}
$$




\section{Appendix B.}

\section{BRST transformations of the four-dimensional model}

$$
\begin{aligned}
& s C_{2 n}^{(0)}=-\sum_{m=-\infty}^{+\infty}\left[C_{2 m+1}^{(0)}, C_{2(n-m)}^{(0)}\right] \\
& s C_{2 n-1}^{(0)}=\sum_{m=-\infty}^{+\infty}\left(\frac{1}{2}\left\{C_{2 m}^{(0)}, C_{2(n-m)}^{(0)}\right\}+\frac{1}{2}\left\{C_{2 m-1}^{(0)}, C_{2(n-m)+1}^{(0)}\right\}\right), \\
& s C_{2 n}^{(1)}=d C_{2 n+1}^{(0)}+\sum_{m=-\infty}^{+\infty}\left(\left[C_{2 m}^{(1)}, C_{2(n-m)+1}^{(0)}\right]-\left\{C_{2 m+1}^{(1)}, C_{2(n-m)}^{(0)}\right\}\right), \\
& s C_{2 n-1}^{(1)}=d C_{2 n}^{(0)}+\sum_{m=-\infty}^{+\infty}\left(\left[C_{2 m}^{(1)}, C_{2(n-m)}^{(0)}\right]-\left\{C_{2 m-1}^{(1)}, C_{2(n-m)+1}^{(0)}\right\}\right), \\
& s C_{2 n}^{(2)}=d C_{2 n+1}^{(1)}+\sum_{m=-\infty}^{+\infty}\left(\left\{C_{2 m}^{(1)}, C_{2(n-m)+1}^{(1)}\right\}-\left[C_{2 m+1}^{(2)}, C_{2(n-m)}^{(0)}\right]-\left[C_{2 m+1}^{(0)}, C_{2(n-m)}^{(2)}\right]\right), \\
& s C_{2 n-1}^{(2)}=d C_{2 n}^{(1)}+\sum_{m=-\infty}^{+\infty}\left(\frac{1}{2}\left\{C_{2 m}^{(1)}, C_{2(n-m)}^{(1)}\right\}+\left\{C_{2 m}^{(0)}, C_{2(n-m)}^{(2)}\right\}-\frac{1}{2}\left\{C_{2 m-1}^{(1)}, C_{2(n-m)+1}^{(1)}\right\}\right. \\
& \left.+\left\{C_{2 m-1}^{(0)}, C_{2(n-m)+1}^{(2)}\right\}\right), \\
& s C_{2 n}^{(3)}=d C_{2 n+1}^{(2)}+\sum_{m=-\infty}^{+\infty}\left(\left[C_{2 m}^{(1)}, C_{2(n-m)}^{(2)}\right]+\left[C_{2 m}^{(3)}, C_{2(n-m)}^{(0)}\right]-\left\{C_{2 m+1}^{(1)}, C_{2(n-m)}^{(2)}\right\}\right. \\
& \left.-\left\{C_{2 m+1}^{(3)}, C_{2(n-m)}^{(0)}\right\}\right) \\
& s C_{2 n-1}^{(3)}=d C_{2 n}^{(2)}+\sum_{m=-\infty}^{+\infty}\left(\left[C_{2 m}^{(1)}, C_{2(n-m)}^{(2)}\right]+\left[C_{2 m}^{(3)}, C_{2(n-m)}^{(0)}\right]+\left\{C_{2 m-1}^{(1)}, C_{2(n-m)+1}^{(2)}\right\}\right. \\
& \left.+\left\{C_{2 m-1}^{(3)}, C_{2(n-m)+1}^{(0)}\right\}\right), \\
& s C_{2 n}^{(4)}=d C_{2 n+1}^{(3)}+\sum_{m=-\infty}^{+\infty}\left(\left\{C_{2 m}^{(1)}, C_{2(n-m)+1}^{(3)}\right\}+\left\{C_{2 m}^{(3)}, C_{2(n-m)+1}^{(1)}\right\}-\left[C_{2 m+1}^{(0)}, C_{2(n-m)}^{(4)}\right]\right. \\
& \left.-\left[C_{2 m+1}^{(2)}, C_{2(n-m)}^{(2)}\right]-\left[C_{2 m+1}^{(4)}, C_{2(n-m)}^{(0)}\right]\right), \\
& s C_{2 n-1}^{(4)}=d C_{2 n}^{(3)}+\sum_{m=-\infty}^{+\infty}\left(\left\{C_{2 m}^{(1)}, C_{2(n-m)}^{(3)}\right\}+\left\{C_{2 m}^{(0)}, C_{2(n-m)}^{(4)}\right\}+\frac{1}{2}\left\{C_{2 m}^{(2)}, C_{2(n-m)}^{(2)}\right\}\right. \\
& \left.-\left\{C_{2 m-1}^{(1)}, C_{2(n-m)+1}^{(3)}\right\}+\left\{C_{2 m-1}^{(0)}, C_{2(n-m)+1}^{(4)}\right\}+\frac{1}{2}\left\{C_{2 m-1}^{(2)}, C_{2(n-m)+1}^{(2)}\right\}\right) .
\end{aligned}
$$

Here the upper indices on $C^{\prime}$ 's indicate the form degrees of the fields: $C^{(2)}=\frac{1}{2} C_{\mu \nu} d x^{\mu} \wedge d x^{\nu}$, for example. 


\section{References}

[1] E. Witten, Nucl. Phys. B311 (1988/89) 46.

[2] N. Kawamoto and Y. Watabiki, Commun. Math. Phys. 144 (1992) 641, Mod. Phys. Lett. A7 (1992) 1137.

[3] N. Kawamoto and Y. Watabiki, Phys. Rev. D45 (1992) 605.

[4] N. Kawamoto and Y. Watabiki, Nucl. Phys. B396 (1993) 326.

[5] M. Henneaux and C. Teitelboim, Quantization of Gauge Systems (Princeton University Press, Princeton, 1992), and references therein.

[6] N. Kawamoto, E. Ozawa and K. Suehiro, Mod. Phys. Lett. A12 (1997) 219.

[7] I.A. Batalin and G.A. Vilkovisky, Phys. Lett. 102B (1981) 27, Phys. Rev. D28 (1983) 2567; Errata: D30 (1984) 508.

[8] E.S. Fradkin and G.A. Vilkovisky, Phys. Lett. 55B (1975) 224.

I.A. Batalin and G.A. Vilkovisky, Phys. Lett. 69B (1977) 309.

E.S. Fradkin and T.E. Fradkina, Phys. Lett. 72B (1978) 343.

I.A. Batalin and E.S. Fradkin, Phys. Lett. 122B (1983) 157, Phys. Lett. 128B (1983) 303 .

[9] L. Brink and J.H. Schwarz, Phys. Lett. 100B (1981) 310.

E.R. Nissimov and S.J. Pacheva, Phys. Lett. 189B (1987) 57.

L. Brink, M. Henneaux and C. Teitelboim, Nucl. Phys. B293 (1987) 505.

U. Lindström, M. Roček, W. Siegel, P. Van Nieuwenhuizen and A.E. Van De Ven, Phys. Lett. 224B (1989) 285.

M.B. Green and C.M. Hull, in Strings 89, eds. R.Arnowitt et al. (World Scientific, 1990).

E. Bergshoeff, R. Kallosh and A. Van Proeyen, Class. Quant. Grav. 9 (1992) 321.

[10] M.B. Green and J.H. Schwarz, Phys. Lett. 136B (1984) 367, Nucl. Phys. B243 (1984) 285. 
R.E. Kallosh, Phys. Lett. 195B (1987) 369.

A.H. Diaz and F. Toppan, Phys. Lett. 211B (1988) 285.

R. Kallosh, W. Troost and A. Van Proeyen, Phys. Lett. 212B (1988) 428.

[11] E. Witten, Nucl. Phys. B268 (1986) 253.

H. Hata, K. Itoh, T. Kugo, H. Kunitomo and K. Ogawa, Phys. Rev. D34 (1986) 2360, Phys. Rev. D35 (1987) 1318.

B. Zwiebach, Nucl. Phys. B390 (1993) 33.

T. Kugo and K. Suehiro, Nucl. Phys. B337 (1990) 434.

C.B. Thorn, Nucl. Phys. B287 (1987) 61.

M. Bochicchio, Phys. Lett. 188B (1987) 330.

[12] N. Kawamoto, K. Suehiro, T. Tsukioka and H. Umetsu, Quantization of infinitely reducible generalized Chern-Simons actions in two dimensions, hep-th/9702172, to be published in Commun. Math. Phys..

[13] L. Baulieu, E. Bergshoeff and E. Sezgin, Nucl. Phys. B307 (1988) 348.

J.C. Wallet, Phys. Lett. 235B (1990) 71.

M. Abud, J.-P. Ader and L. Cappiello, Nuovo Cimento 105A (1992) 1507.

Ö.F. Dayi, Mod. Phys. Lett. A8 (1993) 2087.

L. Baulieu, Field Anti-Field Duality, p-Form Gauge Fields and Topological Field Theories, hep-th/9512026.

[14] J.M.F. Labastida and M. Pernici, Phys. Lett. 212B (1988) 56.

[15] For a review see D. Birmingham, M. Blau, M. Rakowski and G. Thompson, Phys. Rep. 209 (1991) 129, and references therein.

[16] N. Kawamoto, K. Suehiro, T. Tsukioka and H. Umetsu, in preparation.

[17] N. Kawamoto and Y. Watabiki, Commun. Math. Phys. 148 (1992) 169. 